\title{
The Long 3'UTR mRNA of CaMKII Is Essential for Translation-Dependent Plasticity of Spontaneous Release in Drosophila melanogaster
}

\author{
Elena A. Kuklin, ${ }^{1 *}$ Stephen Alkins, ${ }^{1 * ~}$ Baskar Bakthavachalu, ${ }^{2}$ Maria C. Genco, ${ }^{1}$ Indulekha Sudhakaran, ${ }^{2}$ \\ (D). Vijay Raghavan, ${ }^{2}$ Mani Ramaswami, ${ }^{2,3}$ and Leslie C. Griffith ${ }^{1}$ \\ ${ }^{1}$ Department of Biology, Volen National Center for Complex Systems and National Center for Behavioral Genomics, Brandeis University, Waltham, \\ Massachusetts 02454-9110, ${ }^{2}$ National Centre for Biological Sciences, Tata Institute of Fundamental Research, Bangalore 560065, India, and ${ }^{3}$ Trinity College \\ Institute of Neuroscience, School of Genetics and Microbiology and School of Natural Sciences, Trinity College Dublin, Dublin-2, Ireland
}

A null mutation of the Drosophila calcium/calmodulin-dependent protein kinase II gene (CaMKII) was generated using homologous recombination. Null animals survive to larval and pupal stages due to a large maternal contribution of CaMKII mRNA, which consists of a short $3^{\prime}$-untranslated region (UTR) form lacking regulatory elements that guide local translation. The selective loss of the long $3^{\prime} \mathrm{UTR}$ mRNA in CaMKII-null larvae allows us to test its role in plasticity. Development and evoked function of the larval neuromuscular junction are surprisingly normal, but the resting rate of miniature excitatory junctional potentials (mEJPs) is significantly lower in CaMKII mutants. Mutants also lack the ability to increase mEJP rate in response to spaced depolarization, a type of activity-dependent plasticity shown to require both transcription and translation. Consistent with this, overexpression of miR-289 in wild-type animals blocks plasticity of spontaneous release. In addition to the defects in regulation of $\mathrm{mEJP}$ rate, CaMKII protein is largely lost from synapses in the mutant. All phenotypes are non-sex-specific and rescued by a fosmid containing the entire wild-type CaMKII locus, but only viability and CaMKII localization are rescued by genomic fosmids lacking the long 3'UTR. This suggests that synaptic CaMKII accumulates by two distinct mechanisms: local synthesis requiring the long 3'UTR form of CaMKII mRNA and a process that requires zygotic transcription of CaMKII mRNA. The origin of synaptic CaMKII also dictates its functionality. Locally translated CaMKII has a privileged role in regulation of spontaneous release, which cannot be fulfilled by synaptic CaMKII from the other pool.

Key words: activity-dependent plasticity; calcium/calmodulin-dependent protein kinase II; Drosophila; local translation; microRNA; synaptic localization

\section{Significance Statement}

As a regulator of synaptic development and plasticity, CaMKII has important roles in both normal and pathological function of the nervous system. CaMKII shows high conservation between Drosophila and humans, underscoring the usefulness of Drosophila in modeling its function. Drosophila CaMKII-null mutants remain viable throughout development, enabling morphological and electrophysiological characterization. Although the structure of the synapse is normal, maternally contributed CaMKII does not localize to synapses. Zygotic production of CaMKII mRNA with a long 3 ' -untranslated region is necessary for modulating spontaneous neurotransmission in an activity-dependent manner, but not for viability. These data argue that regulation of CaMKII localization and levels by local transcriptional processes is conserved. This is the first demonstration of distinct functions for Drosophila CaMKII mRNA variants.

\section{Introduction}

CaMKII is both ubiquitous and abundant. In mammals, CaMKII constitutes $\sim 1 \%$ of total brain protein (Erondu and Kennedy,
1985), and it is also highly expressed in fly heads (Cho et al., 1991). Unsurprisingly, CaMKII has been shown to have a plethora of important functions in the nervous system, including roles
Bose Fellowship of the Government of India to K.V.R., NCBS Fellows Grant to B.B., CSIR-UGC Fellowship to I.S., and National Institutes of Health Grant P30 NS045713, which supported the Brandeis Imaging Core Facility. We thank Dr. Robert Reenan (Brown University) for advice, encouragement, fly lines, and constructs that allowed us to undertake this homologous recombination project; Dr. Ted Brookings (Q-state Biosciences) for help with analysis methods; Dr. Peter Bronk (Brandeis University) for advice on electrophysiology and analysis; Dr. James Ashely and 
in multiple stages and forms of learning and memory (for review, see Giese and Mizuno, 2013). At the Drosophila neuromuscular junction (NMJ), the roles of CaMKII encompass both development of the synapse and its activity-dependent plasticity. To date, these functions have been revealed using transgenes encoding CaMKII inhibitors or RNAi to decrease kinase activity or activated forms of the kinase to increase activity.

Early studies at the larval NMJ showed that global inhibition of CaMKII with a heat shock-inducible inhibitor peptide transgene (hs-ala lines) increased branching and bouton number (Wang et al., 1994). This same manipulation also increased the amplitude of evoked currents and blocked paired pulse facilitation. Global inhibition of CaMKII was also associated with increased presynaptic excitability (Griffith et al., 1994), whereas expression of constitutively active CaMKII in motor neurons suppressed excitability (Park et al., 2002). Subsequent studies looking at postsynaptic inhibition of CaMKII with both ala peptide and another inhibitor (CaMKIINtide) showed that muscle CaMKII activity could stimulate a retrograde signaling pathway, which increased quantal content without changes in miniature excitatory junctional potential (mEJP) amplitude (Haghighi et al., 2003). The larger quantal content correlated with an increase in morphologically identified release sites and, for high levels of CaMKIINtide expression, an increase in mini-frequency. More recently, presynaptic expression of either ala peptide or CaMKII RNAi was shown to block activity-dependent bouton sprouting (Nesler et al., 2016). The abundance of roles is consistent with the presence of the kinase at high levels on both sides of the NMJ, and in multiple cellular compartments.

The use of genetics to investigate the role of signal transduction molecules in neuronal function has been standard practice for many years in Drosophila. Although Drosophila CaMKII was cloned $>20$ years ago, the location of the gene on heterochromatin-rich chromosome 4 complicated standard mutational approaches. To begin genetic analysis of CaMKII, we generated a null mutation in the CaMKII gene by homologous recombination (Maggert et al., 2008; Staber et al., 2011), inserting two stop codons into the $\mathrm{N}$-terminal coding sequence. In this study, we show that this mutation is completely lethal before adulthood in the homozygous state.

Homozygous mutant animals survive into late larval and pupal stages, due to a large amount of maternally contributed CaMKII mRNA which has a short 3' - untranslated region (UTR) lacking regulatory information, including binding site for miR289 , which are present in the long form. The fact that null animals survive to pupate, and show essentially normal morphological development of the NMJ, implies that maternally-derived short 3'UTR CaMKII is able to support the majority of basic processes. Indeed, transgenic expression of the short 3'UTR form can partially rescue viability, indicating that lack of CaMKII protein is the cause of lethality rather than some critical role of the long 3'UTR form of the mRNA.

Third instar null animals, however, lack the synaptic enrichment of CaMKII seen in wild-type (WT) animals. They also show

Dr. Vivian Budnik (University of Massachusetts Medical School) for advice on setting up the spaced depolarization protocol and the anti-DLG antibody; Dr. Ed Dougherty for help with imaging and NCBS transgenic fly facility; and Krishnan Renganathan for help with generation of CaMKII genomic rescue lines.

The authors declare no competing financial interests.

*E.A.K. and S.A. contributed equally to this work.

Correspondence should be addressed to Dr. Leslie C. Griffith, Department of Biology MS008, Brandeis University, 415 South Street, Waltham, MA 02454-9110. E-mail: griffith@brandeis.edu.

DOI:10.1523/JNEUROSCI.1313-17.2017

Copyright $\odot 2017$ the authors $\quad 0270-6474 / 17 / 3710555-13 \$ 15.00 / 0$ very specific defects in mEJPs and do not exhibit transcription/ translation-dependent plasticity of mEJP frequency. We show that CaMKII derived from newly transcribed mRNA can rescue synaptic localization and viability independent of the $3^{\prime} \mathrm{UTR}$, but plasticity of mEJPs requires the long $3^{\prime} \mathrm{UTR}$ mRNA. Consistent with this, suppression of CaMKII translation in WT animals by overexpression of miR-289 also blocks mEJP plasticity. These results argue that synaptic localization of CaMKII can occur via multiple mechanisms, and that locally translated kinase has a special role in plasticity.

\section{Materials and Methods}

Fly strains. $w^{1118}$; PBac $\left\{\right.$ EGFP-IV\}eIF4G $G^{M 0133}$ (Kyoto DGRC) was used as a fourth chromosome GFP marker. $\left.w{ }^{*}\right] ; D f(4) C 3 / \operatorname{In}(4) c i[D]$, ci[D] pan [ciD] sv[spa-pol] (Bloomington Drosophila Stock Center) contains a deletion that removes the CaMKII locus. OK6-GAL4 and C57-GAL4 were obtained from David Van Vactor (Harvard University). nsyb-GAL4 was obtained from Bloomington Drosophila Stock Center. The UASGFP-CaMKII line was constructed in pUAST (Brand and Perrimon, 1993) using the R3 cDNA reported by Griffith and Greenspan (1993). This cDNA contains $183 \mathrm{bp}$ of $5^{\prime} \mathrm{UTR}$ and $156 \mathrm{bp}$ of $3^{\prime} \mathrm{UTR}$, and is missing the putative miR-289 binding site and the long UTR polyadenylation site.

Homologous recombination. Null mutations in the CaMKII locus were engineered using ends-out recombination following the protocol outlined by Staber et al. (2011). In the exon 2 region of the homology arms, the start ATG (Met) was mutated to TAG (Stop), and an additional stop was added at a Cys codon (see Fig. 1A). DNA was purified using QIAGEN kits and mutations induced using a QuikChange Site-Directed Mutagenesis Kit (Stratagene). Primers were ordered from IDT. Homology arms were inserted into the $\mathrm{P}[\mathrm{w} 25.2]$, and four independent transgenic donor lines were established. A total of 157 recombinants were screened, and 8 lines were isolated. All were homozygous lethal and confirmed to contain the stop codons by sequencing and/or PCR. Experiments were performed with lines 76, 31, and 147 with equivalent results. Data are shown for alleles 76 and 147 .

Primers used are as follows. Mutagenic primers WT cassettes: forward, (LwtSTOP42) 5'-GCCATCGCGATGGCTGCACCAGCAGCCTGTACG CGTTTTTCG-3'; reverse, (RwtSTOP42) 5'-CGAAAAACGCGTACAG GCTGCTGGTGCAGCCATCGCGATGGC-3'. Mutagenic primers stop codoncassettes: forward,(L2stops42)5' -GCCATCGCGtaGGCTGCACCA GCAGCCTGaACGCGTTTTTCG-3'; and reverse, (R2stops42) 5'-CGA AAAACGCGTtCAGGCTGCTGGTGCAGCCtaCGCGATGGC-3'. Validation PCR primers for left arm: (L1971) 5'-TGCCAATTTTAATCGGTA CGCCAGA-3' and ( $p W$-Asc2) $5^{\prime}$-GCTTGGCTGCAGGTCGACTCTAG AGG-3'. Validation primers for right arm: (L9240) 5'-GTCGACGAAGCG CCTCTATTTAT-3' and (R12295) 5'-GGATGAAATGCCGTTGGTTT-3'.

Construction of rescue fosmid lines. Genomic rescues were performed using CaMKII fosmid (FlyFos025846) containing the complete gene with introns, exons, and UTRs expressed under endogenous native regulatory elements. The detailed description of FlyFos025846 construction is described by Ejsmont et al. (2009). In brief, the authors created and catalogued a library of large genomic fragments in an attB sequence containing fosmid vector derived from pCC2fos (Epicenter). From this published library (https://transgeneome.mpi-cbg.de), we obtained the FlyFos025846, which contains $\sim 42.5 \mathrm{~kb}$ of Drosophila melanogaster genomic DNA (sequence data file attached) that contains the entire CaMKII transcript with $\sim 20 \mathrm{~kb}$ of upstream and $\sim 3.3 \mathrm{~kb}$ of downstream genomic sequence. For the $\Delta$ UTR fosmid, 344-2069 bp of the exon 14 sequences were deleted. This preserves the proximal polyadenylation site but deletes everything that follows it, including the distal polyadenylation site and the miR-289 binding elements. Site-specific genome integration for the fosmids was guided by attP landing site and $\phi \mathrm{C} 31$ integrase. Recombinant flies were selected using dominant selectable eye promoter driven dsRed marker (Berghammer et al., 1999).

CaMKII 3'UTR RACE. RNA was prepared from 2-h-old embryos and adult heads (Canton $S$ WT) using a QIAGEN RNeasy kit and 3' Rapid Amplification of cDNA Ends ( $\left.3^{\prime} \mathrm{RACE}\right)$ was performed. RACE-ready 
cDNAs were generated using a SMART RACE cDNA amplification kit (Clontech) with Superscript Reverse Transcriptase III. The CaMKII cDNA prepared with the oligo-dT primers in the kit had an additional SMART sequence (as part of the oligo-dT primer). A gene-specific forward primer close to the $3^{\prime}$ end was used with the universal reverse primer provided in the kit to amplify cDNA ends allowing UTR variations to be identified. Outer and inner forward primers were designed to enable nested PCRs. The primers were designed with a $\mathrm{T}_{\mathrm{m}}$ of $70^{\circ} \mathrm{C}$ so that a touchdown PCR could be done to aid specificity. The PCR products were separated on $0.8 \%-1.2 \%$ agarose gels, then cloned and sequenced to confirm identity.

Immunohistochemistry. To visualize NMJs, third instar larvae were dissected in Modified A solution (in mM as follows: $118 \mathrm{NaCl}, 2 \mathrm{NaOH}, 2$ $\mathrm{KCl}, 4 \mathrm{MgCl}_{2}, 5$ trehalose, 45.5 sucrose, 5 HEPES, adjusted with $1 \mathrm{~N}$ $\mathrm{NaOH}$ to 295 mOsm and pH 7.1-7.2) (Schaefer et al., 2010), then fixed in 4\% PFA for 5-7 min, washed in PBS, and brains cut off. For null mutants, larvae were checked for the presence of GFP to confirm genotype. Body pelts were stained with anti-Dlg (1:50 mouse monoclonal 4F3, Developmental Studies Hybridoma Bank; see Figs. 3 A, B, 6F; or 1:200 rabbit from V. Budnik, see Fig. 4C) and Alexa594-conjugated goat anti-HRP (1:200 Jackson ImmunoResearch Laboratories). Anti-CaMKII 88 was used at 1:500, and anti-Brp (mouse monoclonal nc82) was used at 1:100. Secondary antibodies were AlexaFluor-488, goat anti-rabbit (1:200 Invitrogen), AlexaFluor-488, goat anti-mouse 1:200 (Invitrogen), AlexaFluor-635 goat anti-rabbit IgG (Invitrogen), and AlexaFluor-633 goat anti-mouse (1:200 Invitrogen). Images of the NMJs were taken on a Leica TCS SP5 confocal microscope LAS AF version 2.7.3 at 20× using 488 and $594 \mathrm{~nm}$ lasers sequentially for Dlg (mouse) and HRP, and 488 and 633 lasers for Brp, CaMKII 88, and Dlg.

Embryos were staged and collected to be $13 \mathrm{~h} 30 \mathrm{~min}$ to $14 \mathrm{~h} 30 \mathrm{~min}$ old. They were dechorionated for $3 \mathrm{~min}$ in $50 \%$ bleach and then washed in water. Embryos were fixed in a 4\% PFA and heptane mix 1:1 for $30 \mathrm{~min}$ in a glass vial covered with foil. Devitillinizing was done by replacing the PFA fraction with methanol $+10 \% 0.5 \mathrm{M}$ EGTA and vortexing. Embryos were rinsed $3 \times$ with methanol and washed $3 \times$ with $0.5 \%$ PBT. AntiCaMKII $(88 ; 1: 10,000)$ was applied at $4^{\circ} \mathrm{C}$ for 2 nights; then preparations were washed in PBT, stained in AlexaFluor-635 anti-rabbit IgG (1:200 Invitrogen) at $4^{\circ} \mathrm{C}$ for 1 night, then washed in PBS and mounted using Vectashield (Vector Laboratories). Images of the embryos were taken on Leica TCS SP5 confocal microscope LAS AF version 2.7.0 at $20 \times$ using 488 and $633 \mathrm{~nm}$ lasers sequentially.

For larval brain double staining, third instar larva were dissected in Modified A solution, fixed in 4\% PFA for $10 \mathrm{~min}$, washed in PBS, and then blocked in $10 \%$ NGS for $1 \mathrm{~h}$ at room temperature. Samples were stained in Anti-CaMKII $(88 ; 1: 10,000)$ and anti-Brp mAb nc82 (Developmental Studies Hybridoma Bank, 1:100) at $4^{\circ} \mathrm{C}$ for $1-2$ nights, washed in PBT, incubated in AlexaFluor-555 goat anti-rabbit IgG (1:200 Invitrogen) and Alexa-680 goat anti-mouse (1:200 Invitrogen) for $2 \mathrm{~h}$ at room temperature, then washed in PBT and mounted using Vectashield (Vector Laboratories). Images of the brains were taken sequentially on Leica TCS SP5 confocal microscope LAS AF version 2.7.0 at $20 \times$ using 488, 543 , and $633 \mathrm{~nm}$ lasers sequentially.

For pupal staining, cages of pupae at P6-P7 stages were cut and pupae were fixed in $4 \%$ PFA in $0.2 \%$ PBT for $1 \mathrm{~h}$ on a rotator in a glass vial covered with foil. Pupae were then washed in PBT and dissected in PBS. Samples were washed in PBT, blocked in $10 \%$ NGS, stained in AntiCaMKII $(88 ; 1: 10,000)$ at $4^{\circ} \mathrm{C}$ for 2 nights, washed in PBT, stained in AlexaFluor-635 anti-rabbit $\operatorname{IgG}\left(1: 200\right.$ Invitrogen) at $4^{\circ} \mathrm{C}$ for 1 night, then washed in PBT and mounted using Vectashield (Vector Laboratories). Images of the brains were taken on Leica TCS SP5 confocal microscope LAS AF version 2.7 .0 at $20 \times$ using 488 and $633 \mathrm{~nm}$ lasers sequentially.

Immunoblots. Third instar larvae were dissected in Modified A or Hemolymph-Like saline solution 3.1 (HL3) buffer. Guts, imaginal discs, and brains were removed. Dissected fillets (3 per sample) were frozen on dry ice and then crushed in loading buffer (Laemmli Sample Buffer, Bio-Rad with $5 \% \beta$-mercaptoethanol added) and heated for $10 \mathrm{~min}$. Proteins were separated by SDS-PAGE (NuPAGE Bis-Tris Protein Gels, Invitrogen) and transferred to nitrocellulose membranes (GE Health-
Table 1. Descriptive statistics for CaMKII ${ }^{\text {stopw+ }}$ and sibling control recordings ${ }^{a}$

\begin{tabular}{lcccc}
\hline & $\begin{array}{l}+ \text { I+ sibling } \\
\text { control }\end{array}$ & $N$ & CaMKIItopw + & $N$ \\
\hline Resting membrane potential (mV) & $-62.4 \pm 1.9$ & 9 & $-61.2 \pm 2.3$ & 11 \\
Input resistance $(\mathrm{M} \Omega$ ) & $8.1 \pm 0.5$ & 9 & $7.8 \pm 0.4$ & 11 \\
Quantal content (EJP/mEJP) & $44.3 \pm 2.4$ & 10 & $48.6 \pm 2.6$ & 10 \\
Facilitation/depression index (1st to 4th) & 0.94 & 10 & 0.93 & 10 \\
Facilitation/depression index (1st to 7th) & 0.91 & 10 & 0.93 & 10 \\
Facilitation/depression index (1st to 10th) & 0.88 & 10 & 0.92 & 10 \\
\hline
\end{tabular}

${ }^{a}$ Sibling control and CaMKIItopW+ ${ }^{\text {baseline parameters were compared using one-way ANOVA and Kruskal-Wallis }}$ comparison tests. No significant differences were found. Quantal content was calculated as the ratio between average EJP amplitude and $\mathrm{mEJP}$ amplitude in $\mathrm{mV}$. EJP facilitation/depression indices were obtained by calculating the ratio between respective spikes (spikes 4,7 , and 10 shown in this table) in $5 \mathrm{~V}, 10 \mathrm{~Hz}$ stimulus train and the first evoked potential.

care). Membranes were blocked (Blocking Buffer for Fluorescent Western Blotting, Rockland Immunochemicals) and then incubated with Anti-CaMKII $(88 ; 1: 10,000)$ and anti-actin mAb C4 (1:1000, Millipore). Secondary antibodies were as follows: DyLight 680 mouse and DyLight 800 rabbit Conjugated Pre-Adsorbed antibodies (1:5000, Rockland Immunochemicals). Membranes were scanned on an Odyssey Imager (LI-COR Biosciences). Intensity of bands was calculated using "trim signal" and "background subtraction" features of the software, and the intensity of CaMKII band was normalized to that of actin in the same lane. Statistics were done using Excel.

Determination of lethal phase. CaMKII $I^{\text {stopw }}{ }^{+} / c^{D}$ and $C a M K I I^{W T w+} / c i^{D}$ were crossed to $w$; CantonS WT flies to eliminate the $c i^{D}$ chromosome and replace it with a WT chromosome 4 . Virgins and males of the genotypes CaMKII $I^{\text {stopw+/+ }}$ and CaMKII ${ }^{W T w+/+}$ were collected using the $w+$ marker to follow the mutated fourth chromosome. Crosses were set up for each genotype, and eggs were collected on molasses agar plates.

Electrophysiology. All larval dissections were conducted as in Brent et al. (2009) using third instar larvae in HL3 at a pH of 7.1-7.4, containing the following (in mM): $70 \mathrm{NaCl}, 5 \mathrm{KCl}, 4 \mathrm{MgCl}_{2}, 10 \mathrm{NaHCO}_{3}, 5$ trehalose, 115 sucrose, and 5 HEPES ( $300 \mathrm{Osm}$ ). Body wall muscles were pinned to stretched positions to optimize electrode placement and recording efficacy at NMJ.

mEJPs were recorded intracellularly from muscle of abdominal segments $3-5$ at $20^{\circ} \mathrm{C}-23^{\circ} \mathrm{C}$ in low $\mathrm{Ca}^{2+}\left(0.8 \mathrm{~mm} \mathrm{CaCl}_{2}\right.$ in $\mathrm{HL} 3$ at a $\mathrm{pH}$ of 7.1-7.4). All recordings were analyzed at a sampling rate of $10 \mathrm{kHz}$, for a duration of $2 \mathrm{~min}$. Recordings were discarded if baseline was $> \pm 10 \mathrm{mV}$ of average membrane voltage $(-60 \mathrm{mV})$ or varied by $>5 \mathrm{mV}$ during 2 min recording.

EJPs were recorded intracellularly from muscle of abdominal segments $3-5$ at $20^{\circ} \mathrm{C}-23^{\circ} \mathrm{C}$ in low $\mathrm{Ca}^{2+}\left(0.8 \mathrm{~mm} \mathrm{CaCl}_{2}\right.$ in $\mathrm{HL} 3$ at a $\mathrm{pH}$ of 7.1-7.4), immediately following mEPSP recordings. A suction pipette with a tip opening of $\sim 10 \mu \mathrm{m}$ was used to stimulate the segmental nerve (stimulation duration $=0.1 \mathrm{~ms}$, strength $=5 \mathrm{~V}$, rate $=10 \mathrm{~Hz}$ ). Baseline membrane voltage was recorded for $1 \mathrm{~s}$ before stimulation; total stimulation lasted $1 \mathrm{~s} ; 3 \mathrm{~s}$ of responses was recorded. Recordings were discarded if baseline varied by $>5 \mathrm{mV}$ during stimulation. Only recordings with resting membrane potentials (RMPs) $<-55 \mathrm{mV}$, and input resistances $>5 \mathrm{M} \Omega$ were used for analysis. Table 1 shows descriptive statistics for the recordings.

Spaced depolarization paradigm. Spaced depolarization protocol was adapted from Ataman et al. (2008). Third instar larvae were dissected as previously described without body wall muscle stretching. High $\mathrm{K}^{+}$ (90 mM) HL3 containing the following (in $\mathrm{mm}$ ): $40 \mathrm{NaCl}, 90 \mathrm{KCl}, 20$ $\mathrm{MgCl}_{2}-6 \mathrm{H}_{2} \mathrm{O}, 1.5 \mathrm{CaCl}_{2}, 10 \mathrm{NaCO}_{3}, 5$ sucrose, 5 trehalose, 5 HEPES, $\mathrm{pH}$ 7.2 , was applied to larval preparations in 2, 2, 2, 4, and 6 min pulses, respectively, each separated by a 15 min incubation in normal HL3. Experimental preparations were maintained in normal HL3 for $15 \mathrm{~min}$ after the last high $\mathrm{K}^{+}$pulse and then prepped for recording protocol (as described above). Control larvae underwent all of the same protocols, except perfusion was performed with normal HL3. Actinomycin (5 mM) or cycloheximide $(100 \mathrm{~mm})$ to block transcription or translation, respectively, was included in the normal and high $\mathrm{K}^{+}$saline in indicated experiments.

Experimental design and statistical analyses. For all experiments, $N$ indicates the number of animals. All mEJP and EJP recordings were 


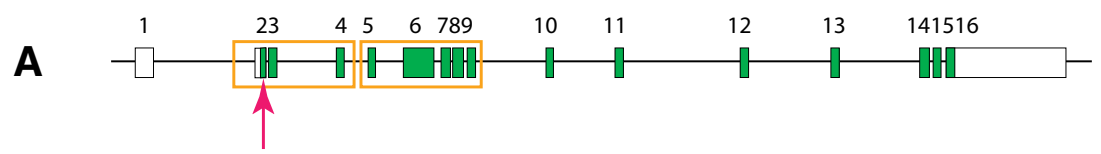

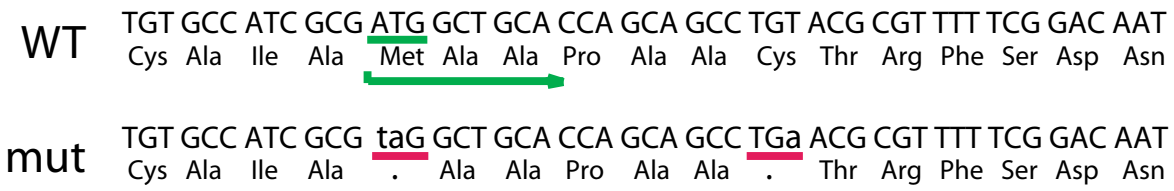

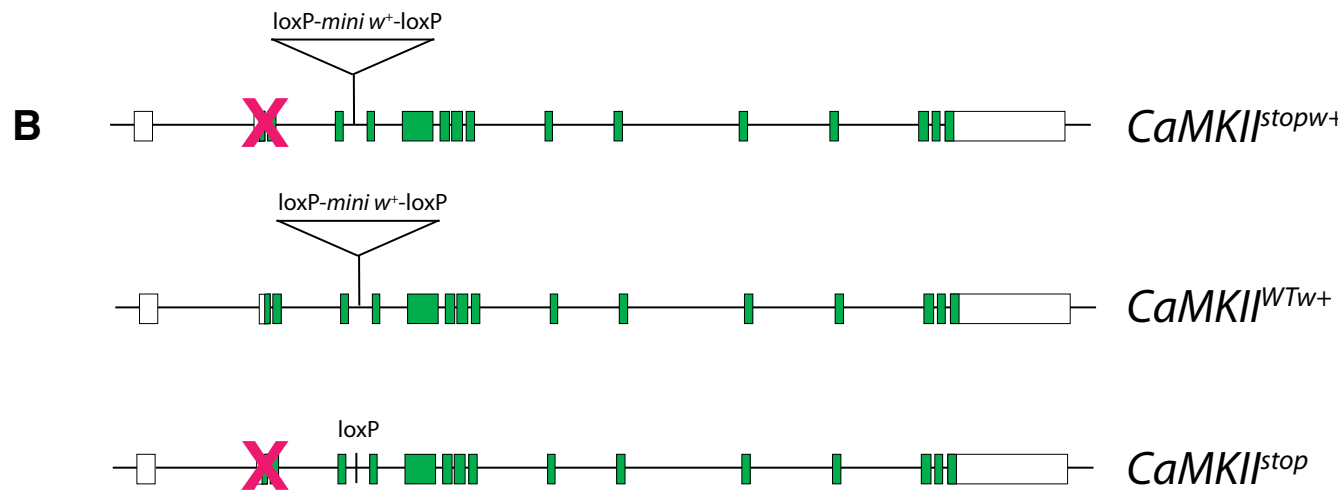

Figure 1. Generation of CaMKIItop null alleles used in this study. A, Strategy for generation of a null mutant. Top, Diagram of the exon organization of the CaMKII locus and the location of recombination arms (gold boxes) and point mutation targets (indicated by red arrow). Green represents coding exons. White represents noncoding. Bottom, Sequence of locus and point mutations induced by homologous recombination. Two stop codons were introduced at Met1 and Cys7. B, Alleles used in this study. The CaMKIItopw+ allele was generated after homologous recombination and retains the mini-white selection cassette, which can be used to follow the engineered chromosome in adults. Induced stop codons are denoted by a red "X." The CaMKIITTw+ control allele was generated by recombination with arms containing the fully WT CaMKII sequence and also contains the mini-white cassette. The CaMKIIItop fully recombined null allele was generated by removing the mini-white cassette using Cre recombinase. It retains a 79 bp loxP site between exons 4 and 5.

analyzed using custom scripts in MATLAB R2016b. Frequency and amplitudes were measured via modified scripts and functions. All analysis code is freely available at https:/github.com/Griffith-Lab/ 2017-Alkins-camkinasemutantpaper. Minimum amplitude for mEJP = $0.4 \mathrm{mV}$. Post hoc Jarque-Bera tests were performed to test for normal distributions of datasets. Subsequent Kruskal-Wallis and two-sample $t$ tests of significance were performed between experimental conditions and genotypes $(\alpha=0.05)$.

Morphological metrics were analyzed by Leica TCS SP5 quantification software, ImageJ Fuji, JMP (SAS), and Excel (Microsoft). Boutons were counted at muscles 6/7 in abdominal segment A2 and A3. Muscles and Dlg-stained regions in boutons were measured from an outlined region of interest. Brp/nc82 puncta were counted from a single $z$ section in the middle of boutons. One-way ANOVA, unpaired two-tailed Student's $t$ test, or $\chi 2$ test was done where appropriate.

Statistics for immunoblot quantification were done using the $t$ test function in Excel.

Electrophysiology figures are presented with mean \pm SEM for clarity. For single comparisons, Student's $t$ test was used, and an asterisk indicates a significant difference between experimental and control groups. Spaced depolarization mEJP recordings were analyzed with SPSS Statistics 24 software (IBM 2016) using a two-way ANOVA with genotype and treatment as main effects. Pairwise comparisons were completed with post hoc Bonferroni correction and Holm-Sidak tests. Significance level for all electrophysiological data statistical tests was $\alpha=0.05$.

\section{Results}

\section{Null mutations at the CaMKII locus are lethal}

Figure $1 A$ shows the strategy we used to generate CaMKII mutants. Ends-out homologous recombination (Rong and Golic, 2000; Staber et al., 2011) was used to introduce two in-frame stop codons at the beginning of the CaMKII coding region in exon 2. Both mutant lines and WT revertant control lines, which had recombined proximal to the mutation, were established. Lines retaining the mini-white gene cassette (which is intronic and efficiently spliced out) and lines where only a 76 bp residual loxP site was present were generated (Fig. 1B).

In the null mutant lines, only heterozygous adults were observed, whereas in WT revertant lines, there was no significant lethality (see Table 3). To determine whether the lethality was due to loss of CaMKII, we generated genomic rescue constructs. The lethality of the null could be rescued by reintroduction of the WT CaMKII genomic region in a fosmid transgene $\left(\mathrm{Fs}^{\mathrm{WT}}\right.$ ) inserted on the third chromosome (Table 2). Rescue was complete, meaning that homozygous null progeny were recovered in a normal Mendelian ratio in the presence of the WT fosmid transgene.

To determine the lethal phase of the null mutation, we did timed embryo collections from crosses between virgin and male flies of the control CaMKII ${ }^{\text {stopw+ }} /+$ genotype and crosses between virgin female and male flies of the null mutant CaMKII ${ }^{\text {stopw+ I+ }}$ genotype. $w+$ lines were used to facilitate identification of the engineered chromosomes. Progression through development was assessed for the progeny. Table 3 shows that there was little embryonic lethality in either cross. Animals from the null/+ cross died in substantial numbers during both the larval and pupal stages, and no null/null adults were recovered. This pattern of lethality suggests that CaMKII is not required for a specific developmental transition before pupation but rather that it has ongoing functions at all stages. The total amount of lethality is consistent with complete loss of homozygous animals, but little dominant lethality in heterozygotes. This may be due to an upregulation of CaMKII in heterozygotes because immunoblots of heterozygotes do not show obvious reductions in protein (E.A.K., 
Table 2. Genomic rescue of CaMKIIstopw+ ${ }^{\text {lethality }}{ }^{a}$

\begin{tabular}{|c|c|c|c|c|c|c|}
\hline \multirow[b]{3}{*}{$\begin{array}{l}\text { Genotype of } \\
\text { F1 Progeny }\end{array}$} & \multicolumn{6}{|l|}{ Parental cross } \\
\hline & \multicolumn{2}{|c|}{ 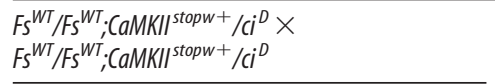 } & \multicolumn{2}{|c|}{ 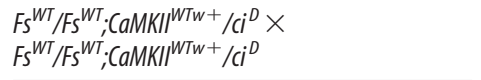 } & \multicolumn{2}{|c|}{ 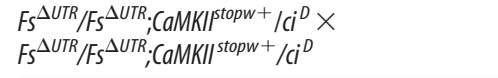 } \\
\hline & $\begin{array}{l}F s^{W T} / F s^{W T} ; \\
\text { CaMKII }^{\text {stopw }} / \text { ci }^{D}\end{array}$ & $\begin{array}{l}F^{W T} / F_{S}{ }^{W T} ; \\
\text { CaMKIII }^{\text {stopw }+} / \text { CaMKII }^{\text {stopw }+}\end{array}$ & 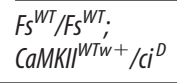 & $\begin{array}{l}F^{W T} / F^{W T} ; \\
\text { CaMKII }^{W T+} / \mathrm{CaMKII}^{W T w+}\end{array}$ & $\begin{array}{l}F s^{\Delta U T R} / F^{\Delta U T R} \\
\text { CaMKII }^{\text {stopw }} / \text { Ci }^{D}\end{array}$ & $\begin{array}{l}F_{5}^{\Delta U T R} / F_{5}^{\Delta U T R} ; \\
\text { CaMKII }^{\Delta t o p w+} / \text { CaMKIII }^{\text {stopw }+}\end{array}$ \\
\hline No. & 119 & 60 & 585 & 290 & 79 & 25 \\
\hline Ratio & 1.98 & & 2.02 & & 3.16 & \\
\hline
\end{tabular}

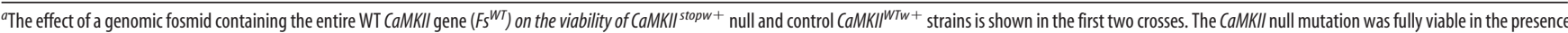
of the $\mathrm{Fs}_{s}{ }^{\mathrm{WT}}$ transgene. The $c^{D}$ marker chromosome is lethal in the homozygous state so that the expected ratio of $c^{D}{ }^{D}$ to non- $c i^{D}$ flies is 2.00 . Two independent $\mathrm{Fs}^{\mathrm{WT}}$ transgenes were tested with equivalent results. $\chi^{2}, p<0.05$. The effects of rescue with a genomic fosmid that does not contain long $3^{\prime} U T R$ sequences $\left(F 5^{\Delta U T R}\right)$ is shown by the third cross. This fosmid provides only partial rescue (ratio of $c i^{D} /$ non- $\left.c i^{D}>2.00\right)$.

Table 3. Lethal phase of the CaMKII ${ }^{\text {stopw+ }}$ mutant $^{a}$

\begin{tabular}{|c|c|c|}
\hline \multirow[b]{2}{*}{ Phase } & \multicolumn{2}{|l|}{$\%$ total mortality } \\
\hline & $\begin{array}{l}\text { CaMKII } I^{\text {stopw }+} /+\times \\
\text { CaMKII }^{\text {stopw }+} /+(\%)\end{array}$ & $\begin{array}{l}\text { CaMKII }^{W T w+} /+\times \\
\text { CaMKII }\end{array}$ \\
\hline Embryo to hatching & 2.9 & 1.7 \\
\hline Hatching to pupation & 16.1 & 3.6 \\
\hline Pupation to adult & 10.1 & 2.0 \\
\hline Total death & 29.1 & 7.3 \\
\hline
\end{tabular}

${ }^{a}$ Progression through development was assessed for the CaMKII ${ }^{\text {stopw }}{ }^{+}$null and control CaMKIIITw+ strains. CaMKII null animals die in both the larval and pupal stages. Death rate in the null strain is consistent with complete preadult lethality for homozygous mutant animals. Collections were done from 16 plates per genotype. $N=1965$ fertilized eggs for CaMKII stopw+ ${ }^{+}$null and 694 for the control CaMKII ${ }^{W T+}$ strain. Full lethality was also observed with an independent allele of CaMKII ${ }^{\text {stopw+ }}$ and in transheterozygotes of the two alleles (data not shown).

data not shown). The nature of this apparent compensation remains to be investigated.

\section{CaMKII-null animals have significant amounts of CaMKII protein due to maternal mRNA}

The ability of null animals to survive into pupation was surprising given the important roles of CaMKII in development. The likely reason for this is that maternally contributed CaMKII mRNA is found in oocytes at high levels (Griffith and Greenspan, 1993). To determine whether this maternal mRNA leads to the presence of CaMKII protein in null animals, we stained the progeny of a cross between virgins and males heterozygous for CaMKII $I^{\text {stopw+ }}$ over a GFP-marked fourth chromosome (see Materials and Methods). Null animals could be unambiguously identified at all developmental stages by their lack of GFP. Figure $2 A$ shows null and WT embryos stained for GFP and CaMKII. There is no quantitative difference in CaMKII levels between genotypes in embryos.

Figure $2 B$ shows a WT $\left(\mathrm{GFP}^{+}\right)$third instar larval brain and a CaMKII-null $\left(\mathrm{GFP}^{-}\right)$brain stained for CaMKII. Levels of the protein are reduced in the null, and there was a significant amount of variability between animals, probably due to differences in the starting amount of maternal mRNA (for clarity, brains with relatively high levels of staining were selected for this and other figures). By pupal stages, average CaMKII levels are even lower than in larvae. P6-P7 brains show low, abnormally distributed, amounts of CaMKII and a grossly altered brain morphology (Fig. 2C). At P13-P15, the few surviving pupae have no CaMKII and the head cavity is liquid-filled, with no discernable brain structures (Fig. 2D). These data indicate that, as maternal mRNA diminishes, CaMKII protein levels fall and postlarval brain development is catastrophically disrupted.

CaMKII distribution is abnormal in null mutants

Although it is clear that overall CaMKII levels fall as development proceeds, Figure $2 B$ also illustrates an apparent change in the distribution of CaMKII, with less neuropil staining in null brains compared with controls. To determine whether this was due to a loss of neuropil integrity or due to a change in the synaptic localization of CaMKII, we costained null third instar larval brains to visualize both CaMKII and Bruchpilot (Brp), an ELKS/CASTS protein that is a presynaptic marker. Neuropil structure in these brains was not obviously disrupted, but there was clearly less CaMKII in that region compared with sibling controls (data not shown). This suggests that maternally derived CaMKII is not effectively localized to synaptic regions in the CNS.

CaMKII is present both presynaptically (Wang et al., 1994; Chapman et al., 1995; Shakiryanova et al., 2011; Nesler et al., 2016) and postsynaptically (Kennedy et al., 1983; Koh et al., 1999) in mammals and Drosophila. To determine whether CaMKII localization defects reflected loss of presynaptic, postsynaptic, or both pools of CaMKII, we examined the NMJ. Costaining of control NMJs with anti-Dlg, a postsynaptic marker, and anti-CaMKII demonstrates the previously observed enrichment of CaMKII in both the presynaptic terminal (middle of boutons) and the subsynaptic reticulum, the Dlg-positive postsynaptic structure of the fly NMJ (Fig. 3A, right panels). Examination of single confocal sections confirmed that CaMKII was present in both presynaptic and postsynaptic compartments in WT animals (data not shown). Significantly, there is almost no synaptic CaMKII in the null animals (Fig. 3A, left panels), despite having easily detectable levels in both the muscle and the motor neuron axon. This indicates that both presynaptically and postsynaptically localized pools of CaMKII are reduced in the mutants, which only have maternal mRNA and lack zygotic transcription of the CaMKII gene.

\section{Third instar larval NMJs are morphologically normal in CaMKII-null animals}

While CaMKII is clearly mislocalized, Figure $3 A$ showed a relatively normal-looking NMJ in the null mutant. To ask whether there were less obvious defects in the developmental program, we assessed the NMJ quantitatively. Previous work using inhibitors of CaMKII had suggested that CaMKII's enzymatic activity has multiple roles in morphological development of the glutamatergic NMJ (Wang et al., 1994; Haghighi et al., 2003; Morimoto et al., 2010; Wang et al., 2011; Timmerman et al., 2013). We stained null and sibling control larval fillets with anti-Dlg to mark the postsynaptic apparatus and with anti-HRP to visualize presynaptic boutons. Figure $3 B$ shows that NMJ structure is grossly normal. Quantitative analysis shows that there are no significant differences in bouton number or postsynaptic area between control and null animals (Fig. 3C,D). There is a slight decrease in muscle area in the null animals compared with siblings (Fig. 3E). These animals have more fragile muscles, and fillets cannot be stretched as easily (E.A.K., unpublished observation), suggesting that they are generally less healthy. Overall, these results imply 
A

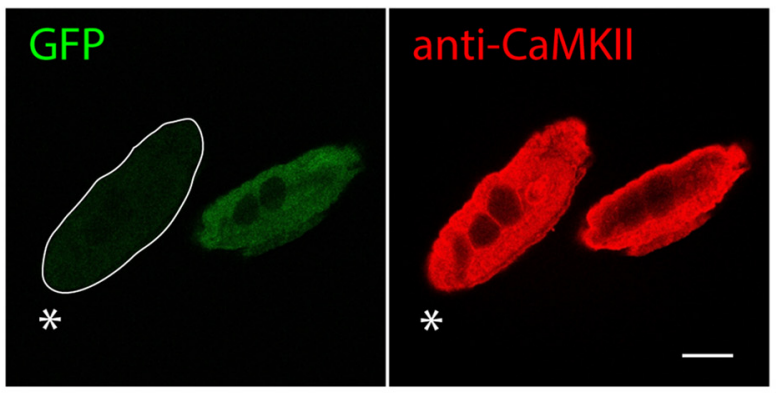

B
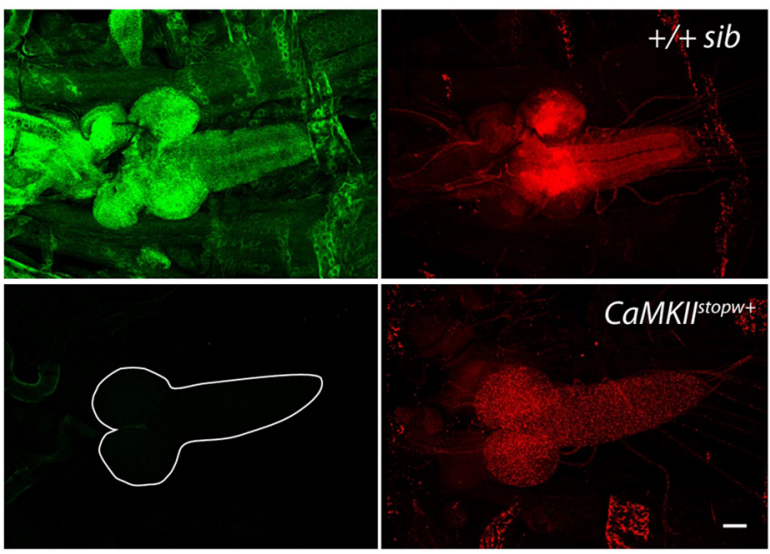

CaMK/1soon

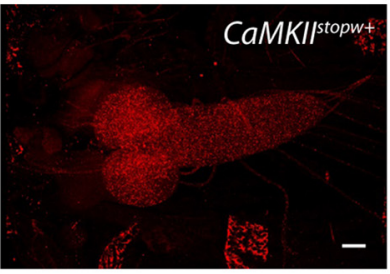

C
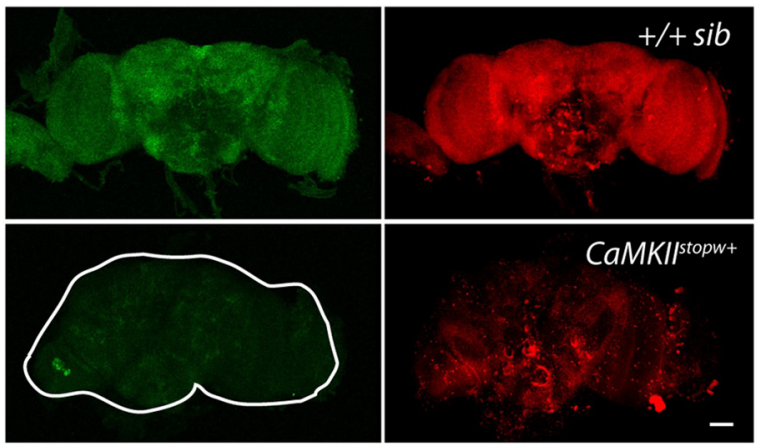

D

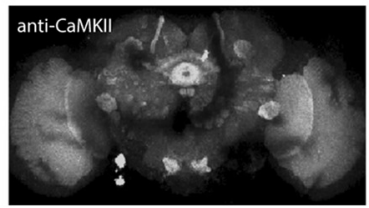

CaMKII ${ }^{W T+}$

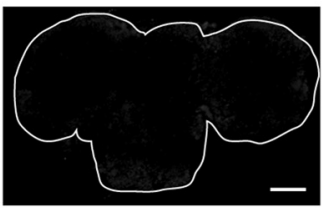

CaMKI/stopw+

Figure 2. CaMKIl is supplied maternally, and levels decline through development. $\boldsymbol{A}$, Embryonic CaMKIIl levels are not reduced in the null mutant. Late-stage embryos from a $w^{1118 . . .}$. PBac $\{$ EGFPIV\}elF4G ${ }^{\text {KM0133 }} /$ CaMKIItopw $^{\text {st }}$ stock were stained for CaMKII with rabbit anti-CaMKII 88 at $1: 10,000$. The presence of the GFP chromosome allows identification of homozygous null, heterozygous, and WT animals from the same cross. Left, GFP visualization with a null animal $\left({ }^{*}\right)$ on the left and a WT animal (sibling control) on the right. Right, The same embryos have similar levels of CaMKII. Scale bar, $100 \mu \mathrm{m}$. B , Third instar larval CaMKII levels are reduced in null mutants and have an abnormal distribution. Top panels, WT sibling control animal with two copies of the GFP chromosome. Bottom panels, Homozygous null animal. GFP visualization (left) and anti-CaMKII 88 staining (right) are shown for both. Samples were dissected, processed, and imaged concurrently. Scale bar, $50 \mu \mathrm{m}$. Levels of CaMKII were variable between individual third instar larvae, likely due to differences in maternal mRNA levels. Images shown are for animals with relatively high levels of CaMKII. C, Stage P6-P7 pupal brains from null animals have a small amount of abnormally distributed CaMKII and appear to be structurally disrupted compared with GFP sibling control. Samples were dissected, processed, and imaged concurrently. Scale bar, $50 \mu \mathrm{m}$. D, Stage P13-P15 pupal brains from CaMKIItopw+ ${ }^{+}$-null animals (right) have no detectable CaMKII compared with CaMKII ${ }^{W T w}+$ sibling controls (left). Brains were stained with mouse monoclonal anti-CaMKII 18 at 1:10,000. Samples were dissected, processed, and imaged concurrently. Scale bar, $50 \mu \mathrm{m}$. that synaptic localization of CaMKII is not required for its roles in the development of the basic structure of the NMJ.

\section{CaMKII-null animals have reduced $\mathrm{mEJP}$ frequency}

The apparently normal morphology of the NMJ was a bit unexpected given the important role of CaMKII's enzymatic activity in structural aspects of development of the synapse. CaMKII has also been shown to be involved in regulation of synaptic transmission at this synapse (Griffith et al., 1994; Wang et al., 1994; Haghighi et al., 2003; Chun-Jen Lin et al., 2011; Wang et al., 2014). To assess the functional development of the NMJ, we performed current-clamp recordings of spontaneous and evoked activity in muscles 6/7. Figure 4 reveals surprisingly normal NMJ function. The response to repetitive stimulation of the NMJ is normal both in terms of the amplitude of EJPs and their dynamics (Fig. 4A) with no changes in facilitation/depression index (Table 1). The amplitude of spontaneous activity is not significantly affected by genotype (Fig. $4 B$, middle), nor is quantal content (data not shown). The one abnormal feature of the CaMKII $I^{\text {stopw }}+$ $\mathrm{NMJ}$ is the mEJP frequency, which is significantly reduced (Fig. $4 B$, right).

Reduced mEJP frequency of CaMKII ${ }^{\text {stopw+ }}$ mutants is not due to a loss of synaptic release sites

The frequency of spontaneous vesicle fusion is controlled by two factors: the number of release sites and the probability of release. At the fly NMJ, each bouton has multiple active zones for neurotransmitter release. Although the number of boutons was not changed in the CaMKII mutant, it was possible that the number of release sites per bouton was reduced. To assess the number of release sites, we examined the localization and amount of Brp, which is a marker for active zones (Wagh et al., 2006). Figure $4 C$ shows boutons double-stained for Dlg and Brp. The number of Brp puncta/ $\mu \mathrm{m}^{2}$ of bouton was not significantly different for null animals compared with sibling controls. This suggests that the reduction in $\mathrm{mEJP}$ frequency is due to a change in the probability of spontaneous release, not the number of available sites.

\section{CaMKII ${ }^{\text {stopw+ }}$ animals are defective for transcription/} translation-dependent plasticity of spontaneous release Neuronal activity is known to regulate evoked release in many systems, but it can also regulate spontaneous release. At the embryonic Drosophila NMJ, four closely spaced $100 \mathrm{~Hz}$ tetani were shown to produce a large (up to $100 \times$ ) increase in the rate of spontaneous release, which lasted for a few minutes (Yoshihara et al., 2005). The same protocol at the third instar NMJ produces a qualitatively similar, but smaller (1.5-2×) increase (Barber et al., 2009; Cho et al., 2015). In contrast to this acute, short-lived regulation of $\mathrm{mEJP}$ rate by a single train of stimuli, long-lasting (hours) increases in mEJP frequency can be produced by applying multiple spaced stimuli with $15 \mathrm{~min}$ intervals. This long-term change in $\mathrm{mEJP}$ rate was shown to require both transcription and translation (Ataman et al., 2008), suggesting that it may depend on mechanisms similar to those that differentiate long-term from short-term memory (Sweatt, 2016).

To determine whether maternally transcribed CaMKII mRNA was sufficient for this type of plasticity, we subjected third instar larval fillets with the CNS intact to a spaced depolarization protocol. Five washes of saline containing $90 \mathrm{mM} \mathrm{K}^{+}$were applied with 15 min interstimulation intervals (schematic in Fig. $5 D$ ). In sibling control larva, this protocol induced an increase in $\mathrm{mEJP}$ frequency of similar magnitude to previous reports (Fig. 5A) (Ataman et al., 2008). The increase in mEJPs in control ani- 

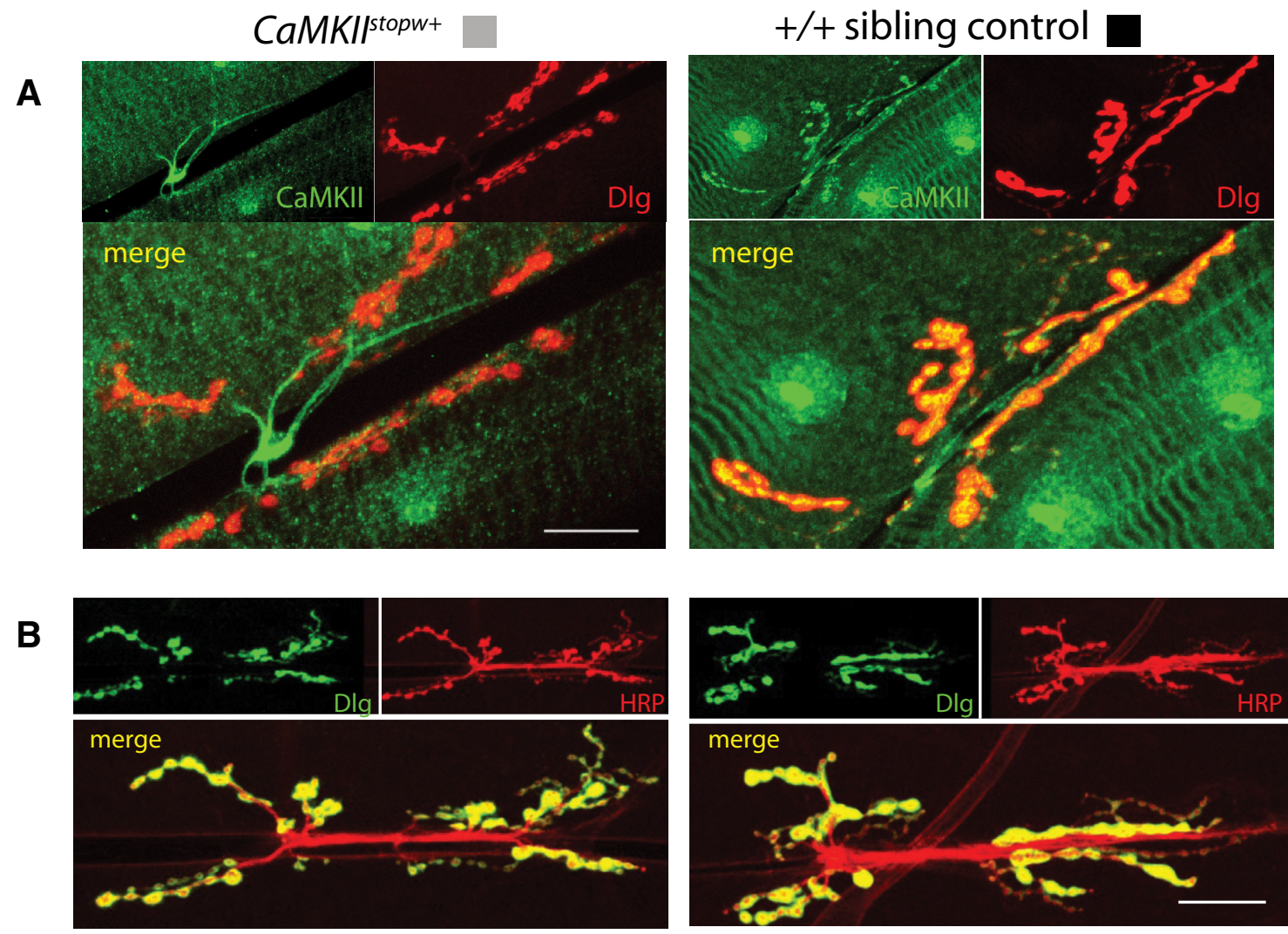

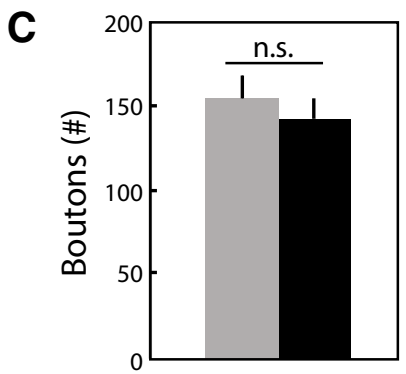

Number of Boutons
D

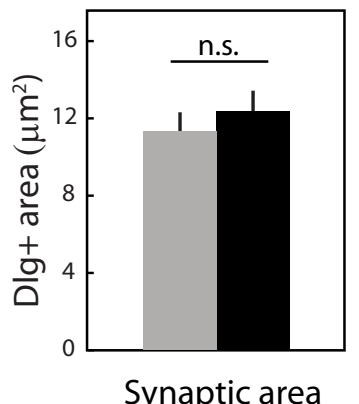

$\mathbf{E}$

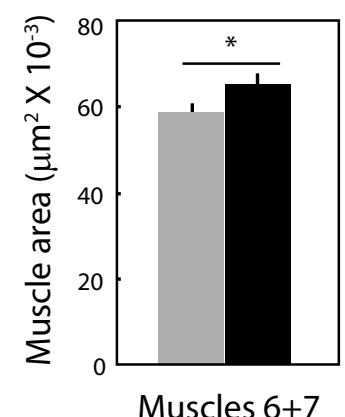

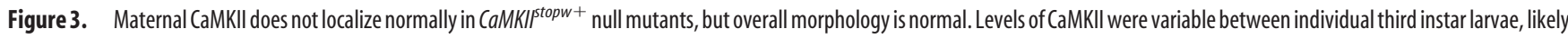
due to differences in maternal mRNA levels. $\boldsymbol{A}, \boldsymbol{B}$, Images are for animals with relatively high levels of CaMKII. $\boldsymbol{A}$, CaMKII mutants lack synaptic CaMKII. Confocal stacks of mutant (left) and WT (right) third instar NMJs stained with anti-CaMKII (1:500 88) and anti-Dlg (1:50 mouse monoclonal 4F3). Samples were dissected, processed, and imaged concurrently with identical settings. Scale bar, $20 \mu \mathrm{m}$. Mutant animals have normal axonal levels of CaMKII but have no synaptic kinase. B, NMJ morphology is normal in CaMKIIftopw+ ${ }^{-n u l l}$ mutants. Third instar larval NMJs were stained with anti-Dlg and anti-HRP, and muscles 6/7 in segment A2 were visualized using a confocal microscope. Left panels, Mutant NMJ. Right panels, Sibling control animal homozygous for the GFP marker chromosome. Scale bar, $25 \mu \mathrm{m}$. C, Bouton number is not altered in CaMKII-null animals. D, Postsynaptic area, as assessed by the measuring the extent of Dlg immunoreactivity, is unchanged in CaMKII-null animals. E, Muscle area is slightly decreased in CaMKII-null animals. The areas of muscles 6 and 7 were assessed using ImageJ Fiji. $N=18$ muscles for CaMKIItopw+ and 16 for WT sibling controls. ${ }^{*} p<0.05$ (Student's $t$ test).

mals was abolished by incubation of the preparation with either $100 \mathrm{~mm}$ cycloheximide or $5 \mathrm{~mm}$ actinomycin. Spaced depolarization was completely unable to induce a change in $\mathrm{mEJP}$ rate in CaMKII $I^{\text {stopw+ }}$ larvae (Fig. 5B). Notably, the basal mini-rate was significantly lower in $C a M K I I^{\text {stopw+ }}{ }^{+}$larvae compared with sibling controls ( $p<0.05$, two-way ANOVA, Bonferroni post hoc test). None of the treatments affected mEJP amplitude in a statistically significant manner ( $p>0.05$, two-way ANOVA, Bonferroni post hoc test; data not shown). These data suggest that new synthesis of CaMKII may be critical to plasticity of spontaneous release.

It was still possible, however, that the failure of null animals to show mEJP plasticity could be due to their overall lower level of CaMKII activity rather than a specific requirement for newly synthesized kinase. To determine whether the absolute level of enzymatic activity was critical for this type of plasticity, we performed the spaced depolarization protocol on ala2 larvae. These animals express a CaMKII inhibitor peptide under control of a heat shock promoter, which results in a significant but incomplete inhibition of CaMKII ( $\sim 25 \%$ decrease), and they have defects in CaMKII-dependent physiology and behaviors, even at room temperature (Griffith et al., 1993). As previously reported (Griffith et al., 1994), these animals had spontaneous synaptic activity, indicating that the ala peptide was expressed (data not shown). Spaced depolarization was able to elicit a significant increase in mEJP frequency in ala2 animals (Fig. 5C). Together, these results demonstrate that a global reduction of CaMKII 
A

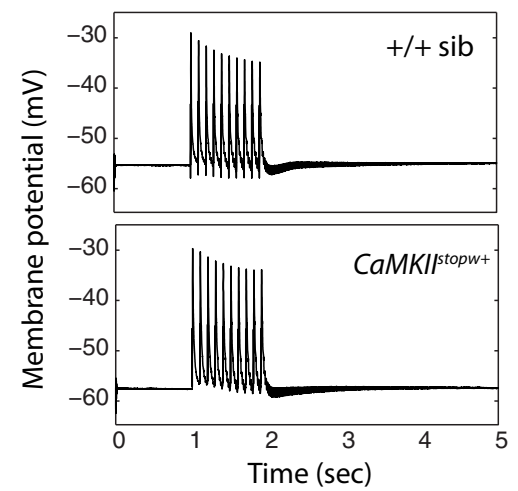

B

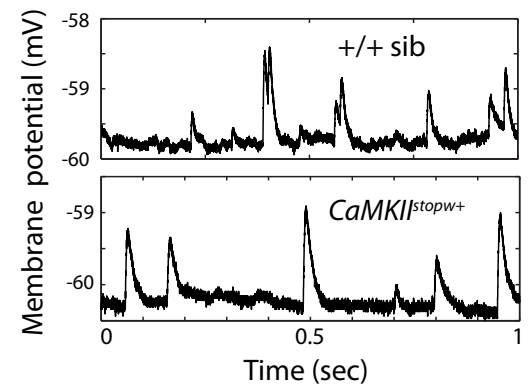

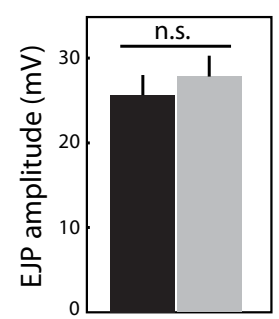

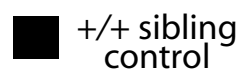

CaMKI/stopw+
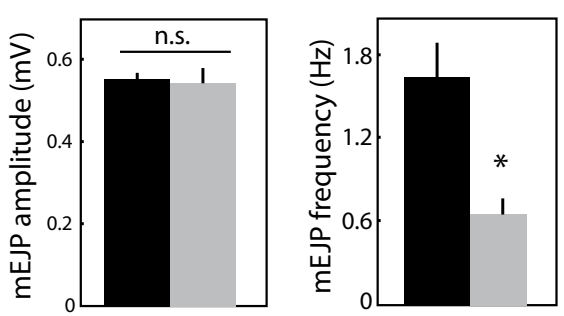

C

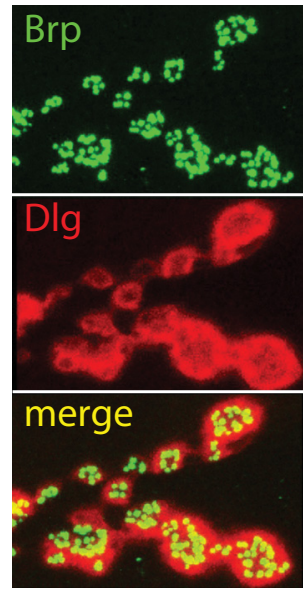

+/+ sibling control

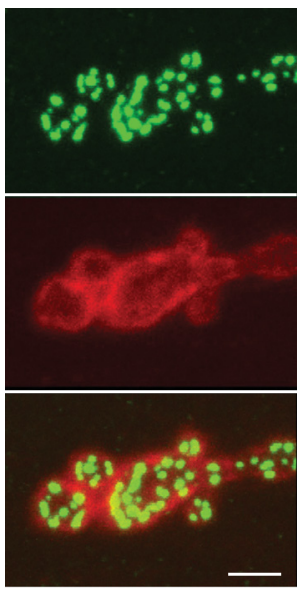

CaMKI/stopw+

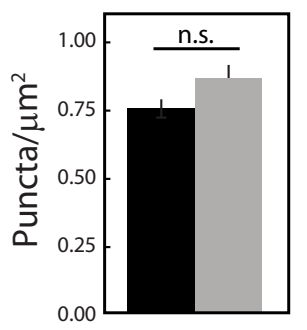

Figure 4. CaMKIItopw ${ }^{+}$-null mutants have decreased mEJP frequency. A, EJPs are normal in the CaMKIPtopw+ mutant. A $1 \mathrm{~s}$ $10 \mathrm{~Hz}$ stimulus delivered to the segmental nerve produces a train of EJPs in both WT and CaMKIItopw+ larvae. Left, Data are average of 50 traces per genotype. Facilitation/depression indices were not affected by genotype (data not shown). Right, Amplitude of the first EJP in the train was not affected by genotype. Data are mean \pm SEM and were analyzed using Student'st test $(p>0.05) ; N=$ 10. $\boldsymbol{B}, \mathrm{mEJP}$ frequency is decreased in the CaMKII mutant. Left, Example traces of mEJPs recorded from muscle 6. Middle, mEJP amplitude was not affected by genotype. Right, mEJP frequency is significantly decreased in CaMKII-null larvae. Average RMP: -62.37 (for sibling control animals); -61.18 (for CaMKIItopw+ animals). Data are mean \pm SEM. ${ }^{*} p<0.05$ (Student's $t$ test). $N=10$. C, Release site number is not changed in the CaMKII mutant. Third instar larval NMJs were stained with mAb nc82, which recognizes Brp, and anti-Dlg to visualize synapses. Left panels, Sibling control NMJ. Right, CaMKIItopw+ mutant NMJ. Scale bar, $5 \mu \mathrm{m}$. The number of nc $82^{+}$puncta per $\mu \mathrm{m}^{2}$ of Dlg staining was assessed and data tested for significance using Student's $t$ test $(p>0.05) . N=16$ for CaMKIItopw+ and 14 for WT sibling controls.

activity is not sufficient to block mEJP plasticity. Thus, the reduction of total CaMKII in the mutant is not likely the source of the mEJP phenotype. The loss of some other feature of zygotically expressed CaMKII is the key factor.

Maternal and zygotic CaMKII mRNAs have different 3'UTRs Although CaMKII levels in our mutant were reduced, and variable between animals, the ability of ala2 animals to increase mEJP rate argued that the failure of mutants to show this plasticity was not linked to the absolute level of CaMKII activity. Interestingly, developmental profiling shows that CaMKII mRNA from very early embryos lacks the long $3^{\prime} \mathrm{UTR}$ found in zygotically transcribed mRNA (Attrill et al., 2016). Using 3' RACE PCR, we confirmed that early embryos have only the short 3'UTR form, whereas adult heads have both short and long forms (Fig. $6 B$ ). In mammals, the 3'UTR of the CAMK2A gene has been shown to regulate localization and translation of the mRNA (Mayford et al., 1996; Steward and Halpain, 1999; Rook et al., 2000), raising the possibility that the fly 3'UTR might be regulatory.

To test the idea that the long $3^{\prime}$ UTR form of mRNA was required for normal development and function, we generated a mutant genomic rescue fosmid ( $\mathrm{Fs}^{\Delta \mathrm{UTR}}$ ) lacking the long UTR sequences (Fig. $6 A$ ). This fosmid still contains the proximal polyadenylation site and is capable of generating a zygotic short 3'UTR mRNA. Larval CaMKII levels in this genotype, as assessed by immunoblotting, were not significantly different from Fs WT (Fig. 6C). This transgene can rescue viability of the null mutant, but not to the level of the WT fosmid. The number of null animals in the presence of Fs ${ }^{\Delta \mathrm{UTR}}$ is $\sim 60 \%$ of the Mendelian expectation (Table 2).

Because CaMKII is expressed in many tissues in Drosophila (Griffith and Greenspan, 1993), we were also interested in whether exclusively neuronal expression of a short 3'UTR form could rescue viability. Driving expression of a CaMKII-GFP cDNA with a truncated 3'UTR in neurons using $n s y b-G A L 4$ also partially rescues viability (data not shown). Together, these results indicate that lethality of the null mutant is primarily due to lack of new synthesis of the CaMKII protein. Production of CaMKII protein in the nervous system alone with a short $3^{\prime}$ UTR mRNA is sufficient for viability, suggesting that the long 3'UTR form has a specialized function. This specialized function likely contributes to the overall health of the animal, but it is not required for progression through metamorphosis.

The long 3'UTR form of CaMKII mRNA is required for basal and regulated spontaneous release

To determine whether the long $3^{\prime}$ UTR contributed to the setting of basal mini-rates and activity-dependent plasticity of minis, we recorded spontaneous activity from $\mathrm{Fs}^{\mathrm{WT}}$ and Fs ${ }^{\mathrm{U} \text { TR }}$ NMJs. Figure $6 D$ shows that basal $\mathrm{mEJP}$ frequency was reduced in animals rescued with $\mathrm{Fs}^{\Delta \mathrm{UTR}}{ }^{\mathrm{A}}$. mEJP rates in animals rescued with the WT 
fosmid were comparable with those found in $+/+$ sibling control animals (compare Fig. $6 D$ and Fig. $4 B$ ), indicating that adding back the normal CaMKII gene can restore mini-rates to WT levels.

To test the role of the long $3^{\prime}$ UTR in plasticity, we applied the spaced depolarization protocol to the two rescue genotypes. Whereas rescue with the WT genomic transgene completely restored plasticity to CaMKII-null animals, addition of a fosmid lacking long 3'UTR sequences did not (Fig. 6E). These data demonstrate that the long $3^{\prime}$ UTR of the CaMKII mRNA is essential for regulation of spontaneous activity.

\section{The long 3'UTR form of CaMKII mRNA is not essential for synaptic localization}

In mammals, concentration of CaMKII at synapses can occur as a result of multiple processes, including local translation of mRNA, which requires UTR sequences (Mayford et al., 1996; Aakalu et al., 2001), and activity-dependent translocation of CaMKII protein, requiring both cytoskeletal and synaptic binding partners (Shen et al., 1998; Shen and Meyer, 1999; Dosemeci et al., 2001). In adult fly brain, 3'UTR sequences appear to be sufficient to target mRNA to dendrites where it can be translated locally, but do not have a role in presynaptic localization of the kinase (Ashraf et al., 2006). In larva, localization of CaMKII to presynaptic terminals does not require the $3^{\prime} \mathrm{UTR}$, but it is used to regulate activity-dependent local translation in that compartment (Nesler et al., 2016). To determine whether the localization defect in the null animals was due to a 3'UTR-dependent process, we stained NMJs from null mutant animals rescued with the full-length CaMKII gene (Fs ${ }^{\mathrm{WT}}$ ) and null animals rescued with the form lacking the long 3'UTR $\left(\mathrm{Fs}^{\mathrm{AUTR}}\right)$ for CaMKII.

Figure $6 F$ shows single confocal sections of third instar NMJs stained with antibodies to Dlg, a postsynaptic marker, and antiCaMKII. In both Fs ${ }^{\text {WT }}$ and Fs ${ }^{\Delta U_{T R}}$ animals, there is clear colocalization. Two examples are shown for each genotype. Figure $6 G$ shows single sections of NMJs stained with antibodies against Brp (a presynaptic marker) and CaMKII. As with the postsynaptic staining, Fs ${ }^{\Delta U T R}$ completely restored presynaptic CaMKII accumulation. The fact that CaMKII can be found both presynaptically and postsynaptically in Fs ${ }^{\Delta \mathrm{UTR}} \mathrm{NMJs}$ suggests that the long $3^{\prime} \mathrm{UTR}$ is not required for synaptic protein localization at the NMJ. Ventral ganglion neuropil CaMKII levels also appeared normal in Fs ${ }^{\Delta U T R}$ animals (data not shown), indicating that larval central neurons also do not require the long $3^{\prime}$ UTR to basally localize CaMKII to synapses or to produce normal levels of CaMKII (Fig. 6C).

These data indicate that the localization of CaMKII protein to larval synapses must have multiple levels of regulation in Drosophila, as it does in mammals. Proper synaptic accumulation in larvae appears to correlate more strongly with new transcription of the CaMKII gene than with mRNA structure. There are a num-
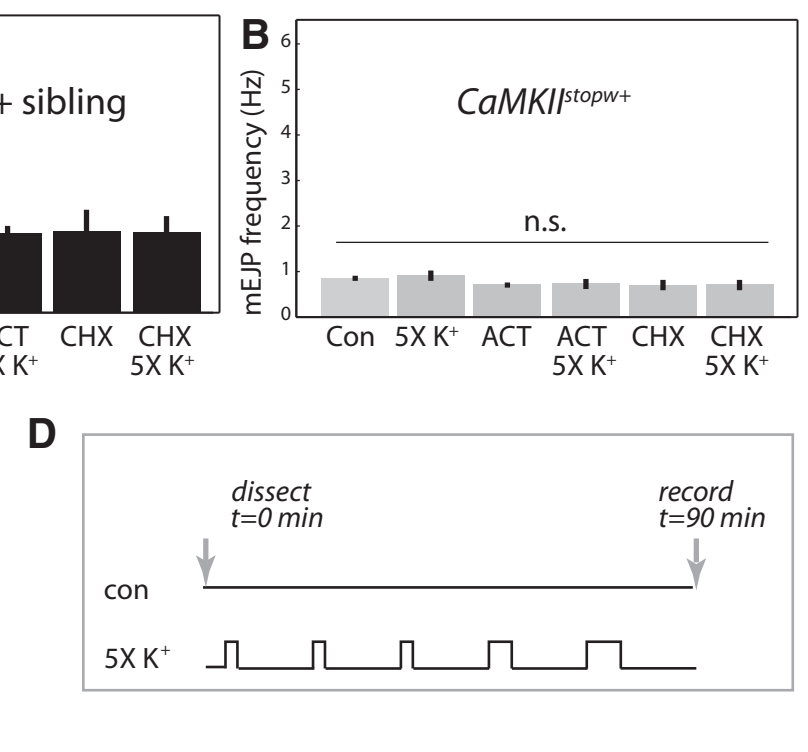

Figure 5. CaMKIStopw ${ }^{+}$-null mutants fail to show activity-dependent increases in mEJP frequency. $\boldsymbol{A}$, Spaced depolarization of WT sibling control animals increases $\mathrm{mEJP}$ frequency in a transcription- and translation-dependent manner. Inclusion of $5 \mathrm{~mm}$ 列 A tests with $\alpha=0.05$. Data are mean \pm SEM. ${ }^{*} p<0.05$, significant difference from all other conditions for that genotype. $m E J P$ amplitude was unchanged by genotype or condition (data not shown).

ber of possible reasons for this, which center around mRNA processing and transport (see Discussion), but it is important to note that we do not yet know where or how the mRNA for CaMKII is localized in the larva, and this information will be critical for formulation of models for CaMKII distribution. In any case, these results strongly imply that simply localizing the kinase protein to the synapse is insufficient to support plasticity of spontaneous release and that some other property of the $3^{\prime} \mathrm{UTR}$ is required.

\section{Regulation of translation of zygotic CaMKII mRNA is critical for plasticity}

Translation is known to be required for plasticity of spontaneous release (Ataman et al., 2008) (Fig. 5A). Recently, it has been shown that activity can induce local increases in CaMKII levels at the NMJ in a translation-dependent manner (Nesler et al., 2016). This is thought to occur by release of CaMKII mRNA from translational suppression by miR-289 (Nesler et al., 2013). Interestingly, the maternal short 3'UTR form of CaMKII mRNA does not contain an miR-289 binding site, suggesting that activitydependent regulation of local translation acts exclusively on zygotically transcribed CaMKII mRNA. To examine the possibility that miR-289 regulation of the long UTR form of CaMKII is required for plasticity of spontaneous release, we overexpressed miR-289 presynaptically using OK6- or c380-GAL4 and postsynaptically using C57-GAL4. In these experiments, all animals have an intact $C a M K I I$ gene.

Figure 7 shows that postsynaptic overexpression of miR-289 completely blocks activity-dependent increases in mini-rate 

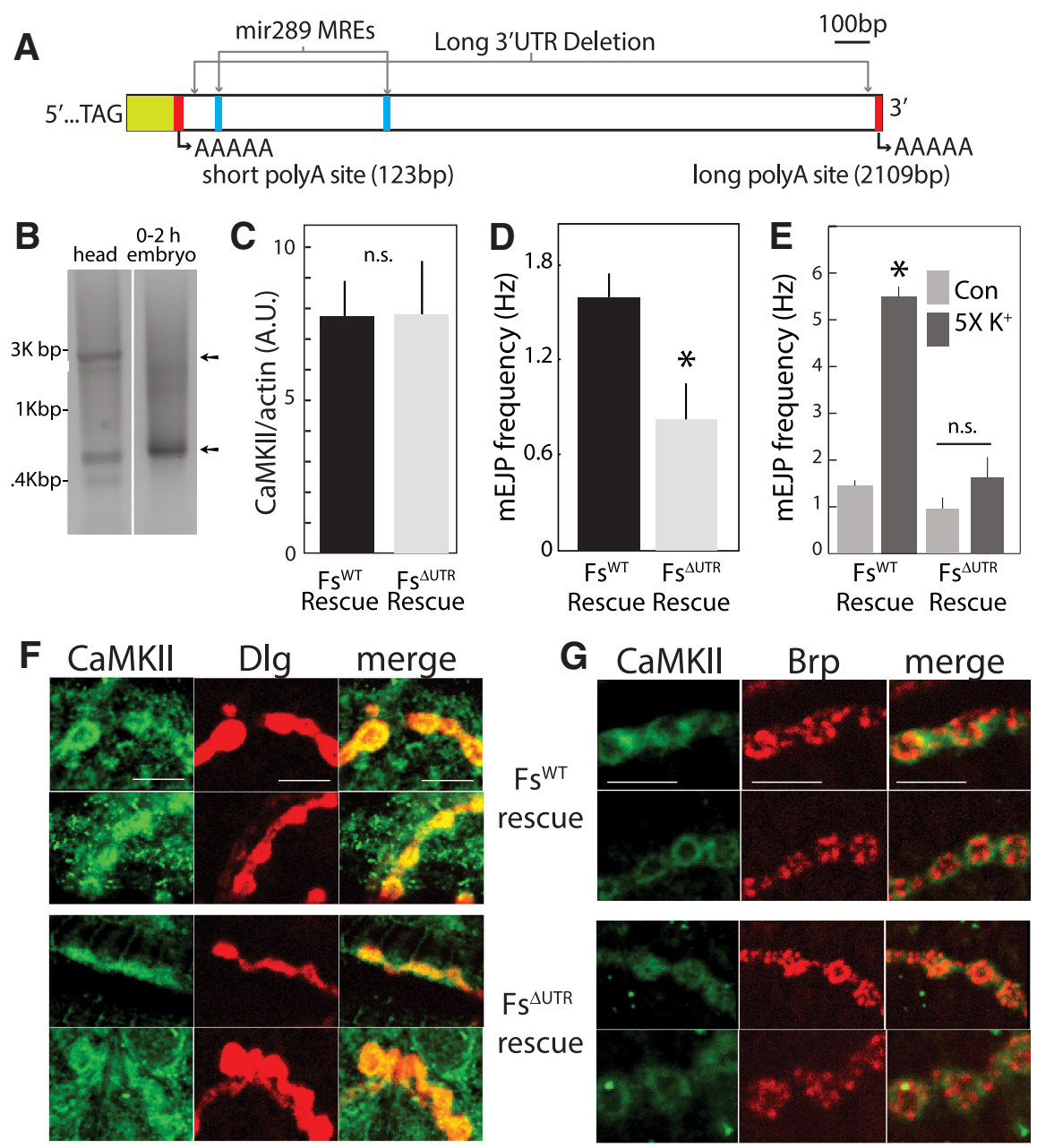

Figure 6. The long $3^{\prime} U T R$ is required for normal mEJP rates but is not required for CaMKII synaptic localization. $A$, Diagram of the $3^{\prime}$ end of the CaMKII gene. $B, 3^{\prime}$ RACE of the CaMKIII $3^{\prime} U T R$ from $0-2$ h embryonic and adult head mRNA. Both a long and a short $3^{\prime}$ UTR are found in adult (arrows), whereas the form containing the long $3^{\prime}$ UTR is not expressed in maternal RNA. Bands were cloned and sequenced to confirm identity. C, CaMKII levels from $\mathrm{Fs}_{\mathrm{ST}}{ }^{\mathrm{WT}}$ and $\mathrm{Fs}_{\mathrm{s}}{ }^{\Delta U \mathrm{TR}}$ larvae are not significantly different. Blots from extracts of 10 independent biological replicates were probed with anti-CaMKII (1:1000, rabbit 88) and anti-actin (1:1000 mouse $\mathrm{mAb}$ (4). Total CaMKII signal was normalized to actin and compared. $p>0.5$ (Student's $t$ test). D, Spontaneous activity was recorded from homozygous CaMKIItopw ${ }^{+}$mutants with chromosome 3 rescue fosmid transgenes containing either the WT CaMKII gene $\left(F_{s}{ }^{\text {WT }}\right.$ ) or a CaMKII gene lacking long $3^{\prime} \mathrm{UTR}$ sequences $\left(\mathrm{Fs}^{\Delta \mathrm{UTR}}\right)$. $\mathrm{Fs}^{\Delta \mathrm{UTR}}$ fails to rescue the CaMKII ${ }^{\text {topw }}{ }^{+} \mathrm{mEJP}$ phenotype (Fig. $4 B$ ). $n=15$ for both $\mathrm{Fs}_{\mathrm{S}}{ }^{\mathrm{WT}}$ and Fs ${ }^{\mathrm{DUTR}}$. Data are mean \pm SEM. ${ }^{*} p<0.05$, significant difference (Student's $t$ test). mEJP amplitude was not significantly different (data not shown). Average RMPs for $\mathrm{Fs}_{s}{ }^{\mathrm{WT}}$ and $\mathrm{Fs}^{{ }^{\Delta U T R}}$ were not significantly different, in $\mathrm{mV}$ as follows: $-63.52,-62.92$, respectively. $E$, A spaced depolarization protocol was applied to Fs ${ }^{\text {WT }}$ and Fs ${ }^{\Delta U T R}$ lines. Fs ${ }^{\Delta U T R}$ fails to rescue mEJP plasticity. $n=6$ for each genotype. Data are mean $\pm S E M .{ }^{*} p<0.05$, significant difference from all other conditions for that genotype (Student's $t$ test). Basal and spaced depolarization-induced mEJP amplitudes were not significantly changed by genotype or condition (data not shown). Average RMPs were not significantly different between control and $5 \times \mathrm{K}^{+}$ conditions for either genotype. In mV after protocol: $-64.17,-63.84$ for $\mathrm{Fs}^{\mathrm{WT}}$ and $\mathrm{Fs}^{\Delta \mathrm{UTR}}$, respectively. F, Postsynaptic CaMKII localizes normally in animals rescued with a fosmid transgene lacking long $3^{\prime}$ UTR sequences. Single confocal sections of mutant animals rescued with a WT fosmid transgene ( $\mathrm{Fs}^{\mathrm{WT}}$, top) and a fosmid lacking long UTR sequences ( $\mathrm{Fs}^{\Delta{ }^{\Delta U T R}}$, bottom). Two examples of each genotype are shown. Third instar NMJs were stained with anti-CaMKII and anti-Dlg. Samples were dissected, processed, and imaged concurrently with identical settings. Scale bar, $10 \mu \mathrm{m}$. G, Presynaptic CaMKII localizes normally in animals rescued with a fosmid transgene lacking long 3'UTR sequences. Single confocal sections of mutant animals rescued with a WT fosmid transgene ( $\left(\mathrm{F}^{\mathrm{WT}}\right.$, top) and a fosmid lacking long UTR sequences ( $\mathrm{Fs}{ }^{\Delta \mathrm{UTR}}$, bottom). Two examples of each genotype are shown. Third instar NMJs were stained with anti-CaMKII and anti-Brp. Samples were dissected, processed, and imaged concurrently with identical settings. Scale bar, $10 \mu \mathrm{m}$.

compared with GAL4/ + and UAS/ + controls ( $p<0.5$, two-way ANOVA with Bonferroni post hoc test). Presynaptic expression of miR-289 with OK6-GAL4 significantly reduces the ability of activity to increase mEJP rate $(p<0.05)$ compared with Canton $S$ WT and GAL4 and UAS controls, whereas c380-GAL4, a very strong driver, completely blocks plasticity. These data are consistent

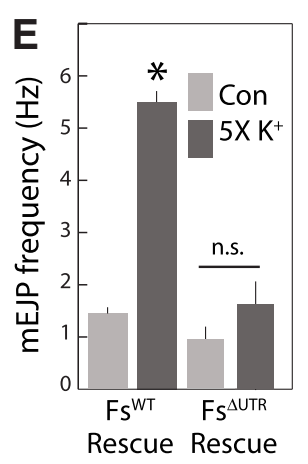

with translational regulation of synaptic CaMKII long 3'UTR mRNA being a critical component of the mechanism of mEJP plasticity.

\section{Discussion}

\section{Maternal CaMKII mRNA allows} initiation of normal development in null larvae but cannot support metamorphosis

Given the numerous and important functions of CaMKII, it is not unexpected that loss of CaMKII is lethal by adulthood. Surprisingly, however, these mutants appear to be fairly normal with respect to the structure and function of the nervous system in larval stages. This is likely because Drosophila embryos receive large amounts of mRNA from maternally derived support cells in the ovary. Because the maternal genotype is CaMKII $I^{\text {stopw+}} /+$, this means that even genetically null oocytes will contain mRNA encoding CaMKII. This mRNA is able to provide normal initial levels of the protein (Fig. 2A); and accordingly, there is no lethality during embryonic development (Table 3 ).

By late larval stages, the amount of CaMKII falls, reflecting either degradation or dilution of maternally encoded kinase. By the time animals reach third instar, functional problems can be seen at the NMJ, and there is significant lethality. The severely reduced CaMKII level in pupal stages blocks the ability to complete metamorphosis. Production of new CaMKII mRNA is clearly necessary for continued viability as the animal enters this stage.

One major difference between the maternal and zygotic mRNAs is that the maternal message has a truncated $3^{\prime}$ UTR (Fig. 6A,B) and lacks sequences known to confer post-transcriptional regulation (Ashraf et al., 2006; Nesler et al., 2016). Interestingly, viability does not seem to require the long $3^{\prime}$ UTR. Animals containing either a rescue fosmid lacking long UTR sequences or expressing a neuronal transgene with a truncated UTR are able to reach adulthood and reproduce. This suggests that producing new protein is sufficient for survival through metamorphosis and that the long UTR form has a specialized role. This underscores the need for future studies to use cell-specific and temporally controlled genetic manipulations of kinase protein and mRNA structure.

The CaMKII-null NMJ phenotype differs from that of animals expressing CaMKII inhibitors

The ability to obtain CaMKII-null third instar larvae allowed us to characterize both the structure of the NMJ and its function in 

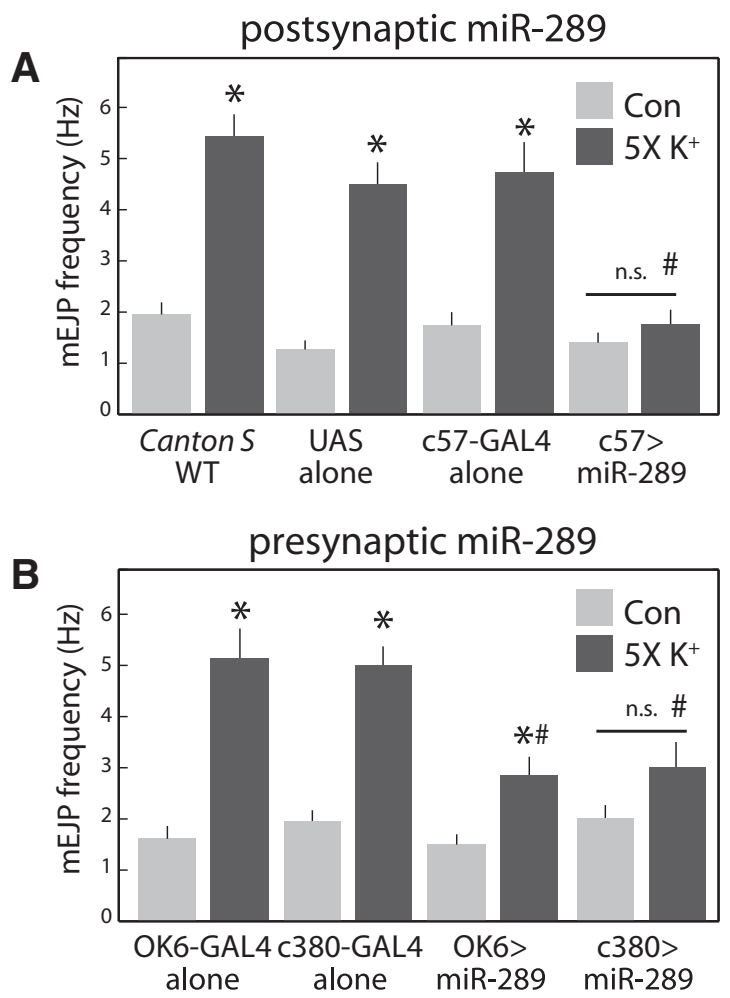

Figure 7. miR-289 overexpression attenuates activity-dependent increases in mEJP frequency. Spaced depolarization protocol was performed with Canton $S$ control animals, and animals overexpressing miR-289 presynaptically (B) in motor neurons (OK6- and C380-GAL4) and postsynaptically $(\boldsymbol{A})$ in muscles (C57-GAL4). RMPs for Canton SCon, Canton S5X K ${ }^{+}$, Pre GAL4 Con, Pre GAL4 $5 \times \mathrm{K}^{+}$, Post GAL4 Con, Post GAL4 $5 \times \mathrm{K}^{+}$, UAS Con, UAS $5 \times \mathrm{K}^{+}$, Pre miR-289 Con, Pre miR-2895 $\times \mathrm{K}^{+}$, Post miR-289 Con, Post miR-2895 $\times \mathrm{K}^{+}$, in mV as follows: -63.87, -62.39, -65.41, -64.68,-63.93,-64.45,-66.46,-64.94,-62.78, -64.56, $-63.77,-66.31$, respectively. Data were analyzed by two-way ANOVA with genotype and treatment as main effects and pairwise comparisons made using Bonferroni post hoc test with $\alpha=0.05$. Data are mean \pm SEM. Control condition was not significantly different across genotypes. *Significant difference from the other condition for that genotype. "Significant difference from WT, UAS, and GAL4 controls for that condition. mEJP amplitude was not significantly changed by genotype or condition (data not shown). RMPs were not significantly different between control and $5 \times \mathrm{K}^{+}$conditions.

the absence of zygotic transcription. What was immediately obvious was that the phenotypes of the null animals did not resemble the phenotypes reported for animals expressing CaMKII inhibitors or RNAi, manipulations that should affect CaMKII activity levels regardless of the mRNA template. Null animals had no obvious morphological defects or changes in excitability or evoked release (Figs. 3, 4), only a decrease in the rate of spontaneous release (Fig. $4 B$ ). In contrast, in animals with strong postsynaptic inhibition of CaMKII, an increase in mini-rate was reported, likely due to an increase in the number of presynaptic release sites (Haghighi et al., 2003). In the CaMKII-null, the number of release sites, as assessed by staining for Brp, is unchanged (Fig. 4C), indicating that the decrease in minis is due to an alteration in release probability. These qualitatively distinct phenotypes suggest that inhibition of CaMKII enzymatic activity is not the same as loss of zygotic transcription on a background of maternally provided kinase.

On the face of it, these differences are surprising because both types of manipulation (transgenic inhibition of CaMKII and loss of new transcription of the gene) should produce animals with reduced CaMKII enzyme activity. What might account for these differences? One possibility is that the absolute levels of CaMKII activity might be different. This would imply that different mag- nitudes of activity loss have qualitatively distinct effects. This possibility seems unlikely, however, given previous findings with the heat shock-driven ala lines where different levels of peptide inhibitor were tested: high and low levels of ala expression did not differ qualitatively, only in severity (Griffith et al., 1994). A second possibility is that the time window in which CaMKII activity is lost is the key difference between the two manipulations. Expression of inhibitors using GAL4 lines that turn on early in development could reduce activity earlier than slow depletion of maternal mRNA does. This would imply that early loss of CaMKII activity has qualitatively different effects than later loss. A third possibility is that RNAi and inhibitor peptides have offtarget effects, perhaps on CaMKI, a poorly studied enzyme in the fly. A fourth possibility, and one we favor, is that inhibition and mutation might be different because they disrupt distinct pools of CaMKII, which are specified by both transcriptional and translational mechanisms.

\section{How is CaMKII from newly transcribed mRNA distinct from that encoded by maternal mRNA?}

Maternal CaMKII mRNA differs in two ways from zygotic mRNA. First, it differs in structure. Drosophila CaMKII has multiple polyadenylation sites and can have either a short or long 3'UTR. Based on publicly available RNA seq datasets (Attrill et al., 2016) and 3' RACE PCR (Fig. 6B), mRNA from 0-2 h embryos (which reflects maternal contribution) contains exclusively the short 3'UTR. The RNA seq data also suggest that it originates from a distinct transcription start site and differs in its $5^{\prime}$ UTR. The second difference is that zygotic mRNA has a different history. Maternal mRNA is synthesized in the nuclei of ovarian nurse cells and never sees the inside of a neuronal nucleus. For CaMKII (Hillebrand et al., 2010) and other mRNAs (Packard et al., 2015), the association with mRNA transport machinery occurs in the nucleus. Newly transcribed nuclear mRNAs therefore have preferential access to the machinery that mediates RNA localization. This machinery can be cell type-specific and change over development, meaning that maternal mRNA, even if it has the correct regulatory sequences, may not be competent to localize correctly. Thus, although maternal and zygotic CaMKII mRNAs encode the same protein, they do not contain the same regulatory information and may not have the same access to localization or processing factors.

How do these two differences influence neuronal structure and function? CaMKII-null mutants have two obvious defects: (1) a decrease in basal and stimulated mEJP rate and (2) a lack of synaptically localized CaMKII. These two deficits appear to be mechanistically distinct. Rescue of synaptic localization was seen with both the WT gene and a fosmid lacking long UTR sequences (Fig. 6F, G). Localization is therefore 3'UTR-independent but appears to require newly transcribed mRNA. Whether this is due to an mRNA-based mechanism (transport of mRNA to synaptic sites and local translation), or whether it is due to preferential transport or diffusion of protein synthesized in the soma from new mRNA templates, will require further investigation.

In contrast to synaptic CaMKII localization, the presence of the long $3^{\prime}$ UTR is absolutely required for establishing a normal basal level of spontaneous release, and for activity-dependent increases in $\mathrm{mEJP}$ rate (Fig. $6 D, E$ ). This plasticity is translationdependent and is suppressed by miR-289 (Fig. 7), which has been previously shown to regulate activity-dependent presynaptic synthesis of CaMKII at the NMJ (Nesler et al., 2016). The partial suppression we see with presynaptic miR-289 could be due to relative expression levels of the mRNA and miR or to a require- 
ment for other regulators. The postsynaptic suppression of plasticity points to involvement of CaMKII-dependent retrograde signaling in spontaneous release. Together, however, these data imply that there is a population of synaptic long $3^{\prime}$ UTR CaMKII mRNA that is locally translated and acts to increase the probability of release. Why newly translated CaMKII is required is unknown, but in rodent neurons, synaptically synthesized CaMKII has preferential access to certain binding partners (Walikonis et al., 2001).

These results also revealed differences between the NMJ and adult olfactory system synapses. In adult projection neurons, the $3^{\prime}$ UTR was required for both localization and activity-dependent regulation of CaMKII translation in dendrites (Ashraf et al., 2006). Further investigation of the mechanisms of RNA and protein localization will be required to resolve these differences, but it is likely that there will be multiple mechanisms for regulation of synaptic CaMKII levels.

\section{Regulation of spontaneous release by local CaMKII}

Activity-dependent synthesis of CaMKII is clearly a critical feature of the enzyme and is conserved across species and developmental stages. Previous work in the adult fly brain has shown that CaMKII mRNA contains sequences that regulate activitydependent translation (Ashraf et al., 2006; Hillebrand et al., 2010; Sudhakaran et al., 2014). Importantly, this was conserved in mammals where 3'UTR sequences in the CAMK2A gene have been shown to drive localization (Mayford et al., 1996; Rook et al., 2000) and activity-dependent translation, although it has been suggested that there may also be a role for $5^{\prime}$ UTR sequences (Aakalu et al., 2001). The fly will provide a powerful model system for understanding how and why CaMKII is targeted to multiple subcellular compartments.

The discovery that local translation of CaMKII is a key driver of plasticity of mini-rate also provides a foothold for obtaining an understanding of this process. Spontaneous release is increasingly being recognized as mechanistically and functionally distinct from evoked release. The regulation of spontaneous release, and even the sites at which it occurs, is separate from action potential-evoked activity (Sara et al., 2005; Sutton and Schuman, 2009; Peled and Isacoff, 2011; Melom et al., 2013; Choi et al., 2014). These miniature events can regulate nuclear gene expression (Wheeler and Cooper, 2004) and local translation (Sutton et al., 2006) and participate in developmental processes, such as circuit wiring (Zucker, 2005; Kavalali et al., 2011). The dependence of activity-dependent plasticity of spontaneous release on local translation of CaMKII on both sides of the synapse suggests that there are complex mechanisms for fine-tuning this important type of synaptic activity.

\section{References}

Aakalu G, Smith WB, Nguyen N, Jiang C, Schuman EM (2001) Dynamic visualization of local protein synthesis in hippocampal neurons. Neuron 30:489-502. CrossRef Medline

Ashraf SI, McLoon AL, Sclarsic SM, Kunes S (2006) Synaptic protein synthesis associated with memory is regulated by the RISC pathway in Drosophila. Cell 124:191-205. CrossRef Medline

Ataman B, Ashley J, Gorczyca M, Ramachandran P, Fouquet W, Sigrist SJ, Budnik V (2008) Rapid activity-dependent modifications in synaptic structure and function require bidirectional Wnt signaling. Neuron 57: 705-718. CrossRef Medline

Attrill H, Falls K, Goodman JL, Millburn GH, Antonazzo G, Rey AJ, Marygold SJ (2016) FlyBase: establishing a Gene Group resource for Drosophila melanogaster. Nucleic Acids Res 44:D786-D792. CrossRef Medline

Barber CF, Jorquera RA, Melom JE, Littleton JT (2009) Postsynaptic regu- lation of synaptic plasticity by synaptotagmin 4 requires both C2 domains. J Cell Biol 187:295-310. CrossRef Medline

Berghammer AJ, Klingler M, Wimmer EA (1999) A universal marker for transgenic insects. Nature 402:370-371. CrossRef Medline

Brand AH, Perrimon N (1993) Targeted gene expression as a means of altering cell fates and generating dominant phenotypes. Development 118: 401-415. Medline

Brent JR, Werner KM, McCabe BD (2009) Drosophila larval NMJ dissection. J Vis Exp 25:1108. CrossRef Medline

Chapman PF, Frenguelli BG, Smith A, Chen CM, Silva AJ (1995) The a-Ca ${ }^{2+} /$ calmodulin kinase II: a bidirectional modulator of plasticity. Neuron 14:591-597. CrossRef Medline

Cho KO, Wall JB, Pugh PC, Ito M, Mueller SA, Kennedy MB (1991) The alpha subunit of type II $\mathrm{Ca}^{2+} /$ calmodulin-dependent protein kinase is highly conserved in Drosophila. Neuron 7:439-450. CrossRef Medline

Cho RW, Buhl LK, Volfson D, Tran A, Li F, Akbergenova Y, Littleton JT (2015) Phosphorylation of complexin by PKA regulates activitydependent spontaneous neurotransmitter release and structural synaptic plasticity. Neuron 88:749-761. CrossRef Medline

Choi BJ, Imlach WL, Jiao W, Wolfram V, Wu Y, Grbic M, Cela C, Baines RA, Nitabach MN, McCabe BD (2014) Miniature neurotransmission regulates Drosophila synaptic structural maturation. Neuron 82:618-634. CrossRef Medline

Chun-Jen Lin C, Summerville JB, Howlett E, Stern M (2011) The metabotropic glutamate receptor activates the lipid kinase PI3K in Drosophila motor neurons through the calcium/calmodulin-dependent protein kinase II and the nonreceptor tyrosine protein kinase DFak. Genetics 188: 601-613. CrossRef Medline

Dosemeci A, Tao-Cheng JH, Vinade L, Winters CA, Pozzo-Miller L, Reese TS (2001) Glutamate-induced transient modification of the postsynaptic density. Proc Natl Acad Sci U S A 98:10428-10432. CrossRef Medline

Ejsmont RK, Sarov M, Winkler S, Lipinski KA, Tomancak P (2009) A toolkit for high-throughput, cross-species gene engineering in Drosophila. Nat Methods 6:435-437. CrossRef Medline

Erondu NE, Kennedy MB (1985) Regional distribution of type II $\mathrm{Ca}^{2+}$ / calmodulin-dependent protein kinase in rat brain. J Neurosci 5:32703277. Medline

Giese KP, Mizuno K (2013) The roles of protein kinases in learning and memory. Learn Mem 20:540-552. CrossRef Medline

Griffith LC, Greenspan RJ (1993) The diversity of calcium/calmodulindependent protein kinase II isoforms in Drosophila is generated by alternative splicing of a single gene. J Neurochem 61:1534-1537. CrossRef Medline

Griffith LC, Verselis LM, Aitken KM, Kyriacou CP, Danho W, Greenspan RJ (1993) Inhibition of calcium/calmodulin-dependent protein kinase in Drosophila disrupts behavioral plasticity. Neuron 10:501-509. CrossRef Medline

Griffith LC, Wang J, Zhong Y, Wu CF, Greenspan RJ (1994) Calcium/ calmodulin-dependent protein kinase II and potassium channel subunit Eag similarly affect plasticity in Drosophila. Proc Natl Acad Sci U S A 91:10044-10048. CrossRef Medline

Haghighi AP, McCabe BD, Fetter RD, Palmer JE, Hom S, Goodman CS (2003) Retrograde control of synaptic transmission by postsynaptic CaMKII at the Drosophila neuromuscular junction. Neuron 39:255-267. CrossRef Medline

Hillebrand J, Pan K, Kokaram A, Barbee S, Parker R, Ramaswami M (2010) The Me31B DEAD-box helicase localizes to postsynaptic foci and regulates expression of a CaMKII reporter mRNA in dendrites of Drosophila olfactory projection neurons. Front Neural Circuits 4:121. CrossRef Medline

Kavalali ET, Chung C, Khvotchev M, Leitz J, Nosyreva E, Raingo J, Ramirez DM (2011) Spontaneous neurotransmission: an independent pathway for neuronal signaling? Physiology (Bethesda) 26:45-53. CrossRef Medline

Kennedy MB, Bennett MK, Erondu NE (1983) Biochemical and immunochemical evidence that the "major postsynaptic density protein" is a subunit of a calmodulin-dependent protein kinase. Proc Natl Acad Sci U S A 80:7357-7361. CrossRef Medline

Koh YH, Popova E, Thomas U, Griffith LC, Budnik V (1999) Regulation of DLG localization at synapses by CaMKII-dependent phosphorylation. Cell 98:353-363. CrossRef Medline

Maggert KA, Gong WJ, Golic KG (2008) Methods for homologous recombination in Drosophila. Methods Mol Biol 420:155-174. CrossRef Medline 
Mayford M, Baranes D, Podsypanina K, Kandel ER (1996) The 3'untranslated region of CaMKII alpha is a cis-acting signal for the localization and translation of mRNA in dendrites. Proc Natl Acad Sci U S A 93:13250-13255. CrossRef Medline

Melom JE, Akbergenova Y, Gavornik JP, Littleton JT (2013) Spontaneous and evoked release are independently regulated at individual active zones. J Neurosci 33:17253-17263. CrossRef Medline

Morimoto T, Nobechi M, Komatsu A, Miyakawa H, Nose A (2010) Subunit-specific and homeostatic regulation of glutamate receptor localization by CaMKII in Drosophila neuromuscular junctions. Neuroscience 165:1284-1292. CrossRef Medline

Nesler KR, Sand RI, Symmes BA, Pradhan SJ, Boin NG, Laun AE, Barbee SA (2013) The miRNA pathway controls rapid changes in activity-dependent synaptic structure at the Drosophila melanogaster neuromuscular junction. PLoS ONE 8:e68385. CrossRef Medline

Nesler KR, Starke EL, Boin NG, Ritz M, Barbee SA (2016) Presynaptic CamKII regulates activity-dependent axon terminal growth. Mol Cell Neurosci 76:33-41. CrossRef Medline

Packard M, Jokhi V, Ding B, Ruiz-Cañada C, Ashley J, Budnik V (2015) Nucleus to synapse Nesprin1 railroad tracks direct synapse maturation through RNA localization. Neuron 86:1015-1028. CrossRef Medline

Park D, Coleman MJ, Hodge JJ, Budnik V, Griffith LC (2002) Regulation of neuronal excitability in Drosophila by constitutively active CaMKII. J Neurobiol 52:24-42. CrossRef Medline

Peled ES, Isacoff EY (2011) Optical quantal analysis of synaptic transmission in wild-type and rab3-mutant Drosophila motor axons. Nat Neurosci 14:519-526. CrossRef Medline

Rong YS, Golic KG (2000) Gene targeting by homologous recombination in Drosophila. Science 288:2013-2018. CrossRef Medline

Rook MS, Lu M, Kosik KS (2000) CaMKIIalpha 3' untranslated regiondirected mRNA translocation in living neurons: visualization by GFP linkage. J Neurosci 20:6385-6393. Medline

Sara Y, Virmani T, Deák F, Liu X, Kavalali ET (2005) An isolated pool of vesicles recycles at rest and drives spontaneous neurotransmission. Neuron 45:563-573. CrossRef Medline

Schaefer JE, Worrell JW, Levine RB (2010) Role of intrinsic properties in Drosophila motoneuron recruitment during fictive crawling. J Neurophysiol 104:1257-1266. CrossRef Medline

Shakiryanova D, Morimoto T, Zhou C, Chouhan AK, Sigrist SJ, Nose A, Macleod GT, Deitcher DL, Levitan ES (2011) Differential control of presynaptic CaMKII activation and translocation to active zones. J Neurosci 31:9093-9100. CrossRef Medline

Shen K, Meyer T (1999) Dynamic control of CaMKII translocation and localization in hippocampal neurons by NMDA receptor stimulation. Science 284:162-166. CrossRef Medline

Shen K, Teruel MN, Subramanian K, Meyer T (1998) CaMKIIb functions as an F-actin targeting module that localizes CaMKIIa/b heterooligomers to dendritic spines. Neuron 21:593-606. CrossRef Medline

Staber CJ, Gell S, Jepson JE, Reenan RA (2011) Perturbing A-to-I RNA editing using genetics and homologous recombination. Methods Mol Biol 718:41-73. CrossRef Medline

Steward O, Halpain S (1999) Lamina-specific synaptic activation causes domain-specific alterations in dendritic immunostaining for MAP2 and CAM kinase II. J Neurosci 19:7834-7845. Medline

Sudhakaran IP, Hillebrand J, Dervan A, Das S, Holohan EE, Hülsmeier J, Sarov M, Parker R, VijayRaghavan K, Ramaswami M (2014) FMRP and Ataxin-2 function together in long-term olfactory habituation and neuronal translational control. Proc Natl Acad Sci U S A 111:E99-E108. CrossRef Medline

Sutton MA, Schuman EM (2009) Partitioning the synaptic landscape: distinct microdomains for spontaneous and spike-triggered neurotransmission. Sci Signal 2:pe19. CrossRef Medline

Sutton MA, Ito HT, Cressy P, Kempf C, Woo JC, Schuman EM (2006) Miniature neurotransmission stabilizes synaptic function via tonic suppression of local dendritic protein synthesis. Cell 125:785-799. CrossRef Medline

Sweatt JD (2016) Neural plasticity and behavior: sixty years of conceptual advances. J Neurochem 139 [Suppl 2]:179-199.

Timmerman C, Suppiah S, Gurudatta BV, Yang J, Banerjee C, Sandstrom DJ, Corces VG, Sanyal S (2013) The Drosophila transcription factor Adf-1 (nalyot) regulates dendrite growth by controlling FasII and Staufen expression downstream of CaMKII and neural activity. J Neurosci 33:1191611931. CrossRef Medline

Wagh DA, Rasse TM, Asan E, Hofbauer A, Schwenkert I, Dürrbeck H, Buchner S, Dabauvalle MC, Schmidt M, Qin G, Wichmann C, Kittel R, Sigrist SJ, Buchner E (2006) Bruchpilot, a protein with homology to ELKS/ CAST, is required for structural integrity and function of synaptic active zones in Drosophila. Neuron 49:833-844. CrossRef Medline

Walikonis RS, Oguni A, Khorosheva EM, Jeng CJ, Asuncion FJ, Kennedy MB (2001) Densin-180 forms a ternary complex with the $\alpha$-subunit of $\mathrm{Ca}^{2+}$ / calmodulin-dependent protein kinase II and $\alpha$-actinin. J Neurosci 21: 423-433. Medline

Wang J, Renger JJ, Griffith LC, Greenspan RJ, Wu CF (1994) Concomitant alterations of physiological and developmental plasticity at CaM kinase II-inhibited synapses in Drosophila. Neuron 13:1373-1384. CrossRef Medline

Wang SJ, Tsai A, Wang M, Yoo S, Kim HY, Yoo B, Chui V, Kisiel M, Stewart B, Parkhouse W, Harden N, Krieger C (2014) Phospho-regulated Drosophila adducin is a determinant of synaptic plasticity in a complex with Dlg and PIP2 at the larval neuromuscular junction. Biol Open 3:1196-1206. CrossRef Medline

Wang S, Yang J, Tsai A, Kuca T, Sanny J, Lee J, Dong K, Harden N, Krieger C (2011) Drosophila adducin regulates Dlg phosphorylation and targeting of Dlg to the synapse and epithelial membrane. Dev Biol 357:392-403. CrossRef Medline

Wheeler DG, Cooper E (2004) Weak synaptic activity induces ongoing signaling to the nucleus that is enhanced by BDNF and suppressed by low levels of nicotine. Mol Cell Neurosci 26:50-62. CrossRef Medline

Yoshihara M, Adolfsen B, Galle KT, Littleton JT (2005) Retrograde signaling by Syt 4 induces presynaptic release and synapse-specific growth. Science 310:858-863. CrossRef Medline

Zucker RS (2005) Minis: whence and wherefore? Neuron 45:482-484. CrossRef Medline 